\title{
ALGORITHMIC SOLUTION OF EXTREMAL DIGRAPH PROBLEMS ${ }^{1}$
}

\author{
BY
}

\author{
W. G. BROWN, P. ERDÖS AND M. SIMONOVITS
}

\begin{abstract}
For a given family $\mathscr{L}$ of digraphs, we study the "extremal" digraphs on $n$ vertices containing no member of $\mathscr{L}$, and having the maximum number of arcs, $\operatorname{ex}(n, \mathscr{L})$. We resolve conjectures concerning the set $\left\{\lim _{n \rightarrow \infty}\left(\operatorname{ex}(n, \mathscr{L}) / n^{2}\right)\right\}$ as $\mathscr{L}$ ranges over all possible families, and describe a "finite" algorithm that can determine, for any $\mathscr{L}$, all matrices $A$ for which a sequence $\{A(n)\}$ of "matrix digraph" is asymptotically extremal $(A(n)$ contains no member of $\mathscr{L}$ and has $\operatorname{ex}(n, \mathscr{L})+o\left(n^{2}\right) \operatorname{arcs}$ as $n \rightarrow \infty$.)
\end{abstract}

\begin{abstract}
REsumk. Pour une famille donnée, $\mathscr{L}$, de digraphes, on étudie les digraphes "extrémaux" à $n$ sommets qui ne contiennent aucun membre de $\mathscr{L}$, et qui possèdent le nombre maximal d'arêtes, ex $(n, \mathscr{L})$. On résolue des conjectures qui concernent l'ensemble $\left\{\lim _{n \rightarrow \infty}\left(\operatorname{ex}(n, \mathscr{L}) / n^{2}\right)\right\}$ ou $\mathscr{L}$ soit une famille quelconque, et on présente un algorithme "fini" qui peut déterminer, pour chaque $\mathscr{L}$, toute matrice $A$ pour laquelle une suite $\{A(n)\}$ de "digraphes matriciels" est extrémale asymptotiquement $\left(A(n)\right.$ ne contient aucun membre de $\mathscr{L}$ et possède ex $(n, \mathscr{L})+o\left(n^{2}\right)$ arêtes lorsque $n \rightarrow \infty$.)
\end{abstract}

1. Introduction. Paul Turán's celebrated theorem $[18,19]$ — which determined, for any $k$, the maximum number of edges in graphs containing no complete $k$-graph and the unique graph realizing that maximum-has led to exact results, and to a developing theory concerning the asymptotic behavior of the extremal numbers and extremal graphs. Brown and Harary [8] and others (cf. [12, 15], etc.) have investigated related problems for directed graphs and multigraphs, with emphasis on exact results. This paper continues a series in which the authors study digraph and multigraph analogues of known asymptotic extremal theorems for graphs. Earlier papers have shown the significance of "matrix digraphs", particularly of digraphs associated with "dense" matrices. The present work resolves conjectures proposed in the first paper of the series [3], concerning the set of attained densities of matrices, and the number of dense matrices realizing a given density. It also refines the main theorem of that first paper, in proving that, for any given family of "prohibited" subdigraphs, there exists an algorithm for determining the set of dense matrices which yield "asymptotically extremal sequences" of digraphs.

We consider digraphs and multigraphs without loops, but with parallel arcs of bounded multiplicity; and, in this introductory section only, simple graphs-i.e. ordinary graphs with neither loops nor multiple edges. If $G$ is a graph, multigraph, or digraph, then $v(G), e(G)$, and $\chi(G)$ respectively denote the numbers of vertices,

Received by the editors March 9, 1984.

1980 Mathematics Subject Classification. Primary 05C35, 05C20; Secondary 05C50.

${ }^{1}$ This research was supported, in part, by an Operating Grant of the Natural Sciences and Engineering Council of Canada held by the first author. 
edges or arcs (counted with multiplicity where applicable), and the chromatic number; a superscript to the symbol denoting a graph, multigraph, or digraph, as in $G^{n}$, always denotes the number of vertices (i.e. $v(G)$ above). (For a general introduction to Extremal Graph Theory, the reader is referred to the book of Bollobás [2] and the survey of Simonovits [17].)

The general problem we wish to consider is the following (where "graph" may also mean "digraph" and "multigraph"):

Problem 1. Given a family $\mathscr{L}$ of graphs (whose members we call prohibited), how many edges can a graph $G^{n}$ of prescribed maximum edge multiplicity possess without containing a subgraph isomorphic to some $L \in \mathscr{L}$ ? The maximum, and the family of "extremal" graphs $G^{n}$ attaining the maximum, will be denoted by $\operatorname{ex}(n, \mathscr{L})$ and $\operatorname{EX}(n, \mathscr{L})$, respectively.

Turán [18, 19] determined $\operatorname{ex}\left(n,\left\{K_{r}\right\}\right)$ and $\operatorname{EX}\left(n,\left\{K_{r}\right\}\right)$. More generally, Erdös and Simonovits [9] proved that when $\mathscr{L}$ is any family of ordinary graphs and $r=\operatorname{Min}\{\chi(L): L \in \mathscr{L}\}, \operatorname{ex}(n, \mathscr{L})=\frac{1}{2}\{1-1 /(r-1)+o(1)\} n^{2}$ as $n \rightarrow \infty$; and that an extremal graph in $\operatorname{EX}(n, \mathscr{L})$ can be obtained from the unique graph in $\operatorname{EX}\left(n,\left\{K_{r}\right\}\right)$ by deleting and adding $o\left(n^{2}\right)$ edges.

Why an "algorithm"? For any family $\mathscr{L}$ of ordinary graphs, (i) $\lim _{n \rightarrow \infty}\left(\operatorname{ex}(n, \mathscr{L}) / n^{2}\right)$, and (ii) the structure of an "asymptotically extremal sequence" of graphs are both known; the limits are all of the form $\frac{1}{2}-1 /(2 r-2)$, where $r$ is a natural number; one "asymptotically extremal sequence" is the "Turán sequence" of extremal graphs for $K_{r}$. For the analogous problem for digraphs the set of limits $\left\{\lim _{n \rightarrow \infty}\left(\operatorname{ex}(n, \mathscr{L}) / n^{2}\right)\right\}$ has infinitely many limit points; and sequences of digraphs other than the obvious analogues of the Turán graphs play a role. Moreover, we do not have a simple characterization, corresponding to the minimum chromatic number criterion for ordinary graphs, of digraphs yielding a specific limit; nor do we have a simple characterization of graphs which arise in asymptotically extremal sequences (although we do have some necessary conditions, cf. Lemma 2 ). Theorem 4 provides an effective algorithm for determining both

$$
\lim _{n \rightarrow \infty}\left(\operatorname{ex}(n, \mathscr{L}) / n^{2}\right)
$$

and a sequence of digraphs "of simple structure" which contain no member of $\mathscr{L}$ and have almost the maximum number of arcs.

Basic definitions appear in $\S \S 2,3$. The main theorems are enunciated in $\S 4$. $\S \S 5$ and 6 are devoted to development of the machinery needed to prove the main results; Theorem 5 is proved in $\S 7$. The pièce de résistance is the Main Lemma, proved in $\S 9$. The main theorems are then proved in $\S \S 10$ and 11 . The final section is devoted to a sketch of analogous results for multigraphs.

2. Digraphs, matrix digraphs. In the sequel we fix a positive integer $q$ and consider only digraphs in which any two vertices are joined by at most $2 q$ arcs, at most $q$ of each orientation. The major results of the present paper pertain to the case $q=1$. The paper should be regarded as a sequel to [3 and 4]; $[6,7]$ were primarily concerned with general $q$. $\mathscr{L}$ always denotes a fixed family of digraphs. 
DEFINITION 1. A sequence $\left\{S_{n}\right\}_{n=1,2, \ldots}$ of digraphs is asymptotically extremal for $\mathscr{L}$ if (i) $S_{n}$ contains no subdigraph $L \in \mathscr{L}$; and (ii) $e\left(S_{n}\right)=\operatorname{ex}\left(v\left(S_{n}\right), \mathscr{L}\right)+$ $o\left(v\left(S_{n}\right)^{2}\right)$ as $n \rightarrow \infty$. Where a sequence is indexed by the numbers of vertices of its members, i.e. where $n=v\left(S_{n}\right)$, sequence members will of ten be represented simply by $S^{n}$.

The objective of our earlier investigations $[3,4,6]$ was to find asymptotically extremal sequences of digraphs having the following "simple" structure: for some positive integer $r$, the vertices can be partitioned into $r$ classes $C_{1}, C_{2}, \ldots, C_{r}$; the number of directed arcs joining a vertex $x$ of $C_{i}$ to a vertex $y$ of $C_{j}$ depends only on $i$ and $j$. This structure is conveniently represented by a matrix, as follows:

Definition 2. (a) Matrix digraphs. Let $A=\left(a_{i j}\right)$ be a square matrix having $r=r(A)$ rows and columns, for which $a_{i j}$ has any value $0,2,4, \ldots, 2 q$ if $i \neq j$; and $a_{i i}=0, \ldots$, or $2 q-1$. Let $\mathbf{a}=\left(a_{1}, a_{2}, \ldots, a_{r}\right)$ be a vector ${ }^{2}$ of nonnegative integers for which

$$
a_{i} \leqslant a_{i i} \leqslant a_{i}+q \leqslant 2 q \text { for } i=1,2, \ldots, r .
$$

For every partition $n=x_{1}+x_{2}+\cdots+x_{r}$ into nonnegative integers we define a digraph $A_{\mathrm{a}}\langle\mathbf{x}\rangle$ or $A_{\mathrm{a}}\left\langle x_{1}, x_{2}, \ldots, x_{r}\right\rangle$ as follows: (i) $n$ vertices are divided into classes $C_{1}, C_{2}, \ldots, C_{r}$ (the $A_{\mathrm{a}}$-classes, or simply $A$-classes), where $\left|C_{i}\right|=x_{i}(i=1,2, \ldots, r)$; (ii) every vertex of $C_{i}$ is joined to every vertex of $C_{j}$ by $\frac{1}{2} a_{i j}$ arcs oriented towards $C_{j}$ $(i, j=1,2, \ldots, r ; i \neq j)$; (iii) each class $C_{i}$ is linearly ordered-whenever $u$ precedes $v$ in this ordering, $u$ and $v$ are joined by $a_{i}$ arcs oriented from $u$ to $v$, and by $a_{i i}-a_{i}$ arcs from $v$ to $u$. The subscript a may be suppressed: $A\langle\mathbf{x}\rangle$, $A\left\langle x_{1}, x_{2}, \ldots, x_{r}\right\rangle$. When $a_{i i}=2 q-1, C_{i}$ is a full class; when $a_{i i}=0$, it is an independent class.

(b) As an algebraic convenience, we admit a null $0 \times 0$ matrix, which we denote by $^{3} \varnothing$. All graphs $\varnothing\langle\mathbf{x}\rangle$ are defined to be empty, having no vertices.

(c) The classes $\mathscr{M}_{r}, \mathscr{M}$. The set of pairs $(A$, a) defined above will be denoted by $\mathscr{M}_{r} . \mathscr{M}=\bigcup_{r=0}^{\infty} \mathscr{M}_{r}$. We may also write $A \in \mathscr{M}_{r}$ or $A \in \mathscr{M}$ to describe an integer matrix $A$ satisfying the conditions of (a), where no vector a is specified.

(d) Matrices $A, A^{\prime} \in \mathscr{M}$ are equivalent (written $A \approx A^{\prime}$ ) if they are equal up to the same permutation of rows and columns. More generally, $(A, \mathbf{a}) \approx\left(A^{\prime}, \mathbf{a}^{\prime}\right)$ if $A$ and $A^{\prime}$ are equal up to the same permutation of rows and columns and a and $\mathbf{a}^{\prime}$ are equal to the same permutation of coordinates.

Definition 3. Optimal matrix digraphs. Any matrix digraph $A_{\mathrm{a}}\langle\mathbf{x}\rangle$ having the maximum number of edges among those for which $\sum_{i} x_{i}=n$ may be denoted by $A_{\mathrm{a}}(n)$ or briefly, by $A(n)$. (Even for fixed a, $A(n)$ may not be uniquely determined, and we often qualify it by an indefinite article.) The vector $\mathbf{x}$ will be called the optimal vector of the $A(n)$.

REMARKS. (1) Remarks following Theorem B (q.v.) justify the structure of Definition 2(a).

\footnotetext{
${ }^{2}$ We use boldface to denote vectors. Also, where convenient, we may interpret a vector $\mathbf{x}$ as a row matrix, which may be transposed.

${ }^{3} \mathrm{~A}$ reader who finds this construct disturbing may dispense with it, and make the appropriate minor changes in several statements and proofs.
} 
(2) If, in $A_{\mathrm{a}}\langle\mathbf{x}\rangle$, we replace any $a_{i}$ by $a_{i i}-a_{i}$, we obtain a digraph having the same structure. We shall therefore assume that

$$
a_{i} \leqslant\left\lfloor\frac{1}{2} a_{i i}\right\rfloor \leqslant q-1 .
$$

In particular, for $q=1$, we may and shall assume that $\mathbf{a}=\mathbf{0}$.

(3) The choice of vector a will not affect the extremal properties under investigation in this paper. We usually speak of "a matrix $A \in \mathscr{M}_{r}$ or $A \in \mathscr{M}^{\text {", }}$, suppressing mention of the vector a. The reader may wish to simplify his first encounter with the concept by assuming, wherever possible, that $\mathbf{a}=\mathbf{0}$.

(4) It is convenient to define a countable digraph $A_{\mathrm{a}}(\infty)$ (briefly $A(\infty)$ ) analogously to $A_{\mathrm{a}}\langle\mathbf{x}\rangle$ : The vertex-set is the union of $r$ countable classes $C_{1}, C_{2}, \ldots, C_{r}$, the members of each class labelled by the natural numbers (and so ordered), with adjacencies determined from $A$ in the obvious way. If a digraph $G$ can be embedded into $A_{\mathrm{a}}(\infty)$, we call it ( $A$, a)-colourable (briefly $A$-colourable.). We emphasize that the type of order imposed on any class is ${ }^{4} \omega$.

(5) The use of twice the multiplicity in the off-diagonal elements is, by design, to simplify certain formulas (cf. (3.1), (3.2)).

(6) In [3] we considered the case $q=1, \mathbf{a}=\mathbf{0}$, using the symbol $A((\mathbf{x}))$ for $A\langle\mathbf{x}\rangle$.

(7) Examples. (i) Any digraph $G$ with adjacency matrix $A$ has structure $(2 A)\langle 1,1, \ldots, 1\rangle$. (ii) Let $B$ be the $1 \times 1$ matrix (1), and let $C=\left(c_{i j}\right)_{i, j=1, \ldots, n}$ be defined by

$$
c_{i j}= \begin{cases}2, & i<j \\ 0, & \text { otherwise }\end{cases}
$$

Then the transitive tournament on $n$ vertices has structure $B\langle n\rangle$ and also $C\langle 1,1, \ldots, 1\rangle$.

3. A connection between matrix graphs and quadratic forms. The number of arcs in $A\langle\mathbf{x}\rangle$ is $^{5}\left(\mathbf{x} A \mathbf{x}^{*}-\sum_{i} a_{i i} x_{i}\right)$. Thus

$$
\mathbf{x} A \mathbf{x}^{*}-(2 q-1) \sum_{i=1}^{r} x_{i} \leqslant 2 e(A\langle\mathbf{x}\rangle) \leqslant \mathbf{x} A \mathbf{x}^{*} \text {. }
$$

Definition 4. (a) Let $(A, \mathbf{a}) \in \mathscr{M}_{r}$. We define the density, $g(A)$, to be $\operatorname{Max}\left\{\mathbf{u} A \mathbf{u}^{*} \mid u_{i} \geqslant 0(i=1,2, \ldots, r) ; u_{1}+u_{2}+\cdots+u_{r}=1\right\}$, i.e. the maximum of the quadratic form associated with $A$ on the standard simplex in $\mathbb{R}^{r}$. $A$ is dense if, for any proper principal submatrix $A^{\prime}, g\left(A^{\prime}\right)<g(A)$; equivalently (for $r>1$ ), if the maximum value is realized only at interior points of the standard simplex. We define $g(\varnothing)=-\infty$.

(b) The subset of $\mathscr{M}_{r}$ consisting of dense matrices (more precisely, of pairs $(A, \mathbf{a})$, where $A$ is dense) will be denoted by $\mathscr{D}_{r}$. $\mathscr{D}$ denotes $\bigcup_{r=0}^{\infty} \mathscr{D}_{r}$.

(c) A vector $\mathbf{u}$ such that $A\langle\mathbf{u}\rangle=g(A)$ is called an optimum vector ${ }^{6}$ of $A$.

\footnotetext{
${ }^{4}$ But cf. Remarks following Lemma 5.

${ }^{5}$ We denote the transpose operation by an asterisk.

${ }^{6}$ This is not to be confused with an optimal vector associated with an optimal matrix digraph.
} 
EXAMPLES FOR $q=1$. (1) The following matrices are dense: $2 J-I$, where $J$ is a matrix of 1 's, and $I$ is the identity matrix; $2 J-2 I$; all matrices of the form

$$
\left(\begin{array}{c|c}
A & 2 J \\
\hline 2 J & B
\end{array}\right)
$$

where $A$ and $B$ are dense.

(2) The following matrices are not dense: all matrices $A$ such that $A+A^{*}$ has a zero off-diagonal element (cf. Lemma 4).

For any $A \in \mathscr{M}$, it follows from (3.1) that [6, (4.2)]

$$
\begin{aligned}
g(A) n^{2} & \geqslant 2 e(A(n)) \\
& =g(A) n^{2}+O(n) \quad \text { as } n \rightarrow \infty .
\end{aligned}
$$

Thus the density is the limit of the average number of arcs joining an unordered pair of vertices in an optimal matrix digraph.

Definition 5. A matrix $A \in \mathscr{M}_{r}$ is asymptotically extremal (or simply extremal) for $\mathscr{L}$ if the sequence $\{A(n)\}$ is asymptotically extremal for $\mathscr{L}$.

Theorem A [3, Theorem 1]. Let $q=1(\mathbf{a}=\mathbf{0})$. For any finite or infinite family $\mathscr{L}$ there exists a dense matrix $A$ which is asymptotically extremal.

We proposed the following conjecture in [6]:

CONJECTURE 1. Theorem A holds without restrictions on $q$ or a: For any family $\mathscr{L}$ there exists $(A, \mathbf{a}) \in \mathscr{D}$ such that $\left\{A_{\mathbf{a}}(n)\right\}$ is asymptotically extremal.

Theorem A is sharp. More precisely, we have announced for $q=1$ [3, Theorem 2], and proved for arbitrary $q$ [6, Theorem 1], the following theorem:

THEOREM B. Let $(A, \mathbf{a}) \in \mathscr{D}$. There exists a finite family $\mathscr{L}$ such that

(i) $\operatorname{EX}(n, \mathscr{L})=\left\{\right.$ all optimal matrix digraphs $\left.A_{\mathrm{a}}(n)\right\}$;

(ii) any asymptotically extremal sequence $\left\{S^{n}\right\}$ for $\mathscr{L}$ can be obtained from $\left\{A_{\mathrm{a}}(n)\right\}$ by deleting and/or adjoining $o\left(n^{2}\right)$ arcs, as $n \rightarrow \infty$; and

(iii) if $\left(A^{\prime}, \mathbf{a}^{\prime}\right) \in \mathscr{D}$, and $\left\{A_{\mathbf{a}^{\prime}}^{\prime}(n)\right\}$ is asymptotically extremal for $\mathscr{L}$, then $\left(A^{\prime}, \mathbf{a}^{\prime}\right) \approx$ $(A, \mathbf{a})$.

REMARK. The structure imposed on the vertices of any one class can be motivated in part from Theorem A-in the case $q=1$. As Theorem A is not known to hold for general $q$, it is possible that some other structure might be "more interesting". However, we do know from Theorem B that, for any $(A, \mathbf{a}) \in \mathscr{D}$, there exists a finite family $\mathscr{L}$ of digraphs for which the sequence $\left\{A_{\mathrm{a}}(n)\right\}$ is extremal, and no other sequence $\left\{A_{\mathbf{a}^{\prime}}^{\prime}(n)\right\}$ is even asymptotically extremal. Thus the case $\mathbf{a}=\mathbf{0}$ alone could not serve to describe the general digraph extremal problem when $q>1$.

4. The main results of the present paper. Theorems 1 and 2 are concerned with the values which the density function may assume. Theorem 3 is an approximation theorem for families of prohibited digraphs; and Theorem 4 describes an algorithm for the determination of "extremal matrices".

For $q=1$, matrices $A=\left(a_{i j}\right)$ such that $a_{i j}+a_{j i} \leqslant 2$ have densities of the form $(1-1 / p) / 2$ (cf. [14]). These densities have only one limit point. For higher multiplicities the situation is more complex (cf. [5]). For example, for $q=1$, the 
attained densities not exceeding $\frac{4}{3}$ are $0, \frac{1}{2}, \frac{2}{3}, \frac{3}{4}, \ldots, 1-1 / r, \ldots ; 1, \frac{8}{7}, \frac{6}{5}, \ldots$, $4 r /(3 r+1), \ldots ; \frac{4}{3}$. From this, and more abundant evidence in the interval from $\frac{4}{3}$ to $\frac{3}{2}$, we were led to formulate conjectures concerning the set of densities and the number of matrices attaining each of them [3, Conjectures 2 and 2*]. These conjectures will be proved in the following theorems.

THEOREM 1. Let $q=1$. For any $\gamma \geqslant 0$ there exist only finitely many dense matrices A such that $g(A)=\gamma$.

Theorem 2. Let $q=1$. The set of attained densities $\{\gamma: \gamma=g(A), A \in \mathscr{D}\}$ is well ordered (under the usual ordering of the reals).

TheOREM 3 (COMPACTNESS). Let $q=1$. For every infinite family $\mathscr{L}$ of prohibited subdigraphs there exists a finite subfamily $\mathscr{L}^{*} \subseteq \mathscr{L}$ for which

$$
\operatorname{ex}(n, \mathscr{L})=\operatorname{ex}\left(n, \mathscr{L}^{*}\right)+o\left(n^{2}\right) \text { as } n \rightarrow \infty
$$

and such that any dense matrix $A$ is asymptotically extremal for $\mathscr{L}$ if and only if it is asymptotically extremal for $\mathscr{L}^{*}$.

Remarks. (1) Suppose $\mathscr{L}^{*} \subseteq \mathscr{L}^{* *} \subseteq \mathscr{L}$ and (4.1) holds. Then $\operatorname{ex}(n, \mathscr{L})=$ $\operatorname{ex}\left(n, \mathscr{L}^{* *}\right)+o\left(n^{2}\right)$ as $n \rightarrow \infty$.

(2) The following weaker theorem has been proved for all $q$.

Theorem C (Continuity) [6, Theorems 3 and 1]. Let $q$ be a positive integer. For every family $\mathscr{L}$ of digraphs, and every $\varepsilon>0$, there exists a finite subfamily $\mathscr{L}^{*} \subseteq \mathscr{L}$ for which, for $n$ sufficiently large,

$$
\operatorname{ex}(n, \mathscr{L}) \leqslant \operatorname{ex}\left(n, \mathscr{L}^{*}\right) \leqslant \operatorname{ex}(n, \mathscr{L})+\varepsilon n^{2} .
$$

The next theorem speaks of an "algorithm", meaning a programme for a "random-access machine (RAM)" (cf. [1]). When the family $\mathscr{L}$ is finite-and also in certain other cases - the "subroutine" spoken of can itself be seen to be finite; then a stronger theorem holds-Theorem $4(\mathrm{~b})$. The input for the RAM is the set $\mathscr{L}$. The output will be a finite set of matrices.

THEOREM 4. Let $q=1$.

(a) Given a subroutine ("oracle") for deciding, for a given family $\mathscr{L}$ and any dense matrix $A$, whether or not some $A(n)$ contains some $L \in \mathscr{L}$, there exists a finite algorithm (independent of $\mathscr{L}$ except that it uses the subroutine) which determines all dense matrices $A$ which are asymptotically extremal for $\mathscr{L}$.

(b) There exists a finite algorithm which determines all dense matrices $A$ for which $\{A(n)\}$ is asymptotically extremal for any given finite family $\mathscr{L}$.

REMARK. We conjecture that Theorems 1-4 remain valid for every $q$. The crucial points where our proofs fail to generalize are Lemma 8, Theorem 5 and the Main Lemma (Lemma 14). The latter may be true for all $q$, but we lack a suitable generalization of Theorem 5 .

Theorems $1-3$ will be proved in $\$ 10$, the algorithm of Theorem 4 in $\$ 11$. 
5. The structure of dense matrices. In $\$ \S 5-7$ we recall, refine, and elaborate on results of [3 and 6] concerning dense matrices, and the procedure we call augmentation.

Definition 6. Let $A \in \mathscr{M} . D(A)$ will denote any principal dense submatrix of $A$ having density $g(A)$. Through use of the indefinite article, we may remind the reader that it need not be unique.

Definition 7. (a) Let $A \in \mathscr{M}_{r} . \hat{A}$ is defined to be $\frac{1}{2}\left(A+A^{*}\right)$.

(b) $\hat{\mathscr{M}}_{r}=\left\{\hat{A}: A \in \mathscr{M}_{r}\right\} . \hat{\mathscr{M}}=\bigcup_{r=0}^{\infty} \hat{\mathscr{M}}_{r}$.

Lemma $1^{7}$ [6, Lemma 2]. Let $A \in \mathscr{D}$. Then $A^{*} \in \mathscr{D}$. Both dense matrices have the same unique optimum vector, i.e. a vector $\mathbf{u}$ with positive coordinates, for which $\mathbf{u} A \mathbf{u}^{*}=\mathbf{u} A^{*} \mathbf{u}^{*}=\mathbf{u} \hat{A} \mathbf{u}^{*}=g(A)=g\left(A^{*}\right)$; namely the unique positive solution $\mathbf{u}$ of the equations ${ }^{8}$

$$
\hat{A} \mathbf{u}^{*}=g(A) \mathbf{e}^{*}, \quad(\mathbf{u}, \mathbf{e})=1,
$$

where $\mathbf{e}=(1,1, \ldots, 1)$.

Lemma 2. Let $A \in \mathscr{D}_{r}$ have optimum vector $\mathbf{u}, r>1$. Then

(a) $\hat{A}$ is nonsingular;

(b) $g(A)=1 / \mathbf{e} \hat{A}^{-1} \mathbf{e}^{*}$;

(c) $\mathbf{u}=\left(1 / \mathbf{e} \hat{A}^{-1} \mathbf{e}\right) \mathbf{e} \hat{A}^{-1}$; thus the coordinates of $\mathbf{u}$ are all rational.

Proof. (a) Suppose $\mathbf{v}$ is a nonzero vector such that $\hat{A} \mathbf{v}^{*}=\mathbf{0}^{*}$. Let $\alpha$ be any real number, sufficiently small that all coordinates of $\mathbf{u}+\alpha \mathbf{v}$ are positive; define $\mathbf{w}=(1+\alpha(\mathbf{v}, \mathbf{e}))^{-1}(\mathbf{u}+\alpha \mathbf{v})$. Then $\mathbf{w} A^{*} \mathbf{w}^{*}=(1+\alpha(\mathbf{v}, \mathbf{e}))^{-2} g(A)$. If $(\mathbf{v}, \mathbf{e})=0$, this contradicts the uniqueness of the optimum vector $\mathbf{u}$. Since $r>1, g(A)>0$; if $(\mathbf{v}, \mathbf{e}) \neq 0, \alpha$ taken with the opposite sign to $(\mathbf{v}, \mathbf{e})$ yields $\mathbf{w} A \mathbf{w}^{*}>g(A)$, contradicting the maximality of $g(A)$.

(b) Solving (5.1), we obtain $\mathbf{u}^{*}=g(A) \hat{A}^{-1} \mathbf{e}^{*}$. Then $1=(\mathbf{u}, \mathbf{e})=g(A) \mathbf{e} \hat{A}^{-1} \mathbf{e}^{*}$.

(c) $\mathbf{u}=g(A) \mathbf{e}\left[\hat{A}^{-1}\right]^{*}=\left(1 / \mathbf{e} \hat{A}^{-1} \mathbf{e}^{*}\right) \mathbf{e} \hat{A}^{-1}$.

REMARKS. (1) Lemma 2 readily yields a finite algorithm to determine whether or not a given matrix is dense.

(2) There exist nonsingular matrices $\hat{A} \in \hat{\mathscr{M}}$ such that the vector $\mathbf{e} \hat{A}^{-1} / \mathbf{e} \hat{A}^{-1} \mathbf{e}^{*}$ has positive coordinates, but that $\hat{A}$ is not dense. Consider, for example, the matrix

$$
\begin{aligned}
& A=\left[\begin{array}{llll}
0 & 4 & 6 & 4 \\
0 & 0 & 2 & 6 \\
0 & 0 & 1 & 4 \\
0 & 0 & 0 & 1
\end{array}\right] \text { where } q=3 . \\
& \hat{A}^{-1}=\frac{1}{9}\left[\begin{array}{rrrr}
2 & -7 & 11 & -5 \\
-7 & 11 & -16 & 13 \\
11 & -16 & 20 & -14 \\
-5 & 13 & -14 & 8
\end{array}\right] .
\end{aligned}
$$

The submatrix obtained by deleting the second row and column of $A$ has density $\frac{11}{6}$, which is greater than $\frac{9}{5}=1 / \mathbf{e} \hat{A}^{-1} \mathbf{e}^{*}$.

\footnotetext{
${ }^{7}$ To be generalized in $\$ 12$.

${ }^{8}$ We denote the inner product of $\mathbf{x}$ and $\mathbf{y}$ by $(\mathbf{x}, \mathbf{y})$. Thus, following our earlier conventions, $(\mathbf{x}, \mathbf{y})=\mathrm{xy}^{*}$.
} 
(3) A dense matrix $A$ need not be nonsingular. Consider, for example, the matrices which are twice the adjacency matrix of a transitive tournament.

(4) If $\hat{A}$ is nonsingular, but $A$ is not dense, $g(A)>1 / \mathbf{e} \hat{A}^{-1} \mathbf{e}^{*}$. (Evaluate the quadratic form at $\left(1 / \mathbf{e} \hat{A}^{-1} \mathbf{e}^{*}\right) \mathbf{e} \hat{A}^{-1}$.)

Lemma 3 [3, p. 84]. Let $A \in \mathscr{D}_{r}$.

(a) Valencies of vertices in $A(n)$ differ by at most $2 q$.

(b) Valencies of vertices of any $A(n)$ are $g(A) n+O(1)$ as $n \rightarrow \infty$.

(c) Let $A\left\langle\mathbf{x}^{(n)}\right\rangle$ be an optimal digraph $A(n)(n=0,1, \ldots)$. Then $\lim _{n \rightarrow \infty} \frac{1}{n} x^{(n)}=\mathbf{u}$, the optimum vector characterized in (5.1).

Lemma 4 [Generalizing 3, Lemma 1]. Let $A \in \mathscr{D}_{r}(r>1)$. Then $a_{i j}+a_{j i}>a_{i i}$ $+a_{j j}(i, j=1,2, \ldots, r ; i \neq j)$.

Proof. Without limiting generality, take $i=1, j=2$; let $\mathbf{u}=\left(u_{1}, u_{2}, \ldots, u_{r}\right)$ be the optimum vector of $A$, and define $\mathbf{v}=(1,-1,0,0, \ldots, 0), \mathbf{w}=\mathbf{u}+u_{1} \mathbf{v}$. Since $(\mathbf{w}, \mathbf{e})=1$, and $\mathbf{w}$ lies in the boundary of the standard simplex in $\mathbb{R}^{r}, \mathbf{u} A \mathbf{u}^{*}-\mathbf{w} A \mathbf{w}^{*}$ $>0$; hence $u_{1}^{2} \mathbf{v} A \mathbf{v}^{*}<2 u_{1} \mathbf{v} \hat{A} \mathbf{u}^{*}=2 u_{1} g(A) \mathbf{v e} \mathbf{e}^{*}=0$ by (5.1). But $\mathbf{v} A \mathbf{v}^{*}=a_{11}+a_{22}$ $-a_{12}-a_{21}$.

REMARK. Lemma 4 implies that the only dense matrices $A$ of density less than 1 have $A=J-I$.

Definition 8. Let $(A, \mathbf{a}) \in \mathscr{D}$, and let $\left\{G^{n}\right\}$ be a sequence of digraphs. Define $l_{n}=\max \left\{l: A_{\mathrm{a}}(l) \subseteq G^{n}\right\} . A$ is weakly contained in $\left\{G^{n}\right\}$ if $\limsup _{n \rightarrow \infty} l_{n}=\infty$. $(A, \mathbf{a})$ is strongly contained in $\left\{G^{n}\right\}$ if $\lim _{n \rightarrow \infty} l_{n}=\infty$.

Definition 9. Let $(A, \mathbf{a}) \in \mathscr{M}_{r}$ and $\left(A^{\prime}, \mathbf{a}^{\prime}\right) \in \mathscr{M}_{r^{\prime}}$.

(a) We write $(A, \mathbf{a}) \uparrow\left(A^{\prime}, \mathbf{a}^{\prime}\right)$, or briefly $A \uparrow A^{\prime}$, when, for infinitely many $m$, $A_{\mathbf{a}}(m) \subseteq A_{\mathbf{a}^{\prime}}^{\prime}(\infty)$.

(b) An embedding of $A_{\mathrm{a}}\langle\mathbf{x}\rangle$ into $A_{\mathbf{a}^{\prime}}^{\prime}\left\langle\mathbf{x}^{\prime}\right\rangle$ is canonical if there exists a mapping $f$ : $\{1,2, \ldots, r\} \rightarrow\left\{1,2, \ldots, r^{\prime}\right\}$ such that all vertices of any class $C_{i}$ of $A_{\mathrm{a}}\langle\mathbf{x}\rangle$ are together embedded into class $C_{f(i)}^{\prime}$ of $A_{\mathbf{a}^{\prime}}^{\prime}\left\langle\mathbf{x}^{\prime}\right\rangle . f$ is said to induce the canonical embedding. Where the same $f$ induces canonical embeddings of every $A_{\mathrm{a}}\langle\mathbf{x}\rangle$ into suitable $A_{\mathbf{a}^{\prime}}^{\prime}\left\langle\mathbf{x}^{\prime}\right\rangle$ (or $\left.A_{\mathbf{a}^{\prime}}^{\prime}(\infty)\right)$, we call $f$ canonical, and write $f:(A, \mathbf{a}) \uparrow\left(A^{\prime}, \mathbf{a}^{\prime}\right)$, or simply $f: A \uparrow A^{\prime}$.

Lemma 5. Let $(A, \mathbf{a}) \in \mathscr{M}_{r}$ and $\left(A^{\prime}, \mathbf{a}^{\prime}\right) \in \mathscr{M}_{r^{\prime}}$.

(a) $(A, \mathbf{a}) \uparrow\left(A^{\prime}, \mathbf{a}^{\prime}\right)$ if and only if there exists a canonical mapping $f:(A, \mathbf{a}) \uparrow\left(A^{\prime}, \mathbf{a}^{\prime}\right)$.

(b) For a canonical mapping $f:(A, \mathbf{a}) \uparrow\left(A^{\prime}, \mathbf{a}^{\prime}\right)$ the following inequalities hold:

$$
\begin{aligned}
& \text { For any } i, a_{i} \leqslant a_{f(i)}^{\prime}, a_{i i} \leqslant a_{f(i), f(i)}^{\prime} . \\
& \text { When } f(i) \neq f(j), a_{i j} \leqslant a_{f(i), f(j)}^{\prime} . \\
& \text { When } i \neq j, \text { but } f(i)=f(j), \\
& \qquad \operatorname{Min}\left(a_{i j}, a_{j i}\right) \leqslant 2 a_{f(i)}^{\prime}, a_{i j}+a_{j i} \leqslant 2 a_{f(i), f(j)}^{\prime} .
\end{aligned}
$$

Conversely, the truth of these inequalities implies that $f$ induces canonical embeddings.

(c) The following conditions are equivalent: (i) $\left(A\right.$, a) $\uparrow\left(A^{\prime}, \mathbf{a}^{\prime}\right)$; (ii) $(A$, a) is strongly contained in any sequence $\left\{A_{\mathbf{a}^{\prime}}^{\prime}(n)\right\}$; (iii) $A$ is weakly contained in any sequence $\left\{A_{\mathbf{a}^{\prime}}^{\prime}(n)\right\}$. 
(d) If $A \uparrow A^{\prime}, g(A) \leqslant g\left(A^{\prime}\right)$.

(e) If $A$ and $A^{\prime}$ are dense, $g(A)=g\left(A^{\prime}\right)$, and $A \uparrow A^{\prime}$, then $A \approx A^{\prime}$. Thus the density function is strictly order-preserving on the set of equivalence classes of $\mathscr{D}$ under $\approx$, partially ordered by $\uparrow$.

Proof. (a) Suppose $(A, \mathbf{a}) \uparrow\left(A^{\prime}, \mathbf{a}^{\prime}\right)$. For sufficiently large $m$ every embedding of $A_{\mathrm{a}}(m)$ into $A_{\mathrm{a}^{\prime}}^{\prime}(\infty)$ carries at least 2 vertices of $C_{i}$ of $A_{\mathrm{a}}(m)$ into some class $C_{j_{i}}^{\prime}$ of $A_{\mathrm{a}^{\prime}}^{\prime}(\infty)$; define $f(i)=j_{i}(i=1,2, \ldots, r)$. The converse is obvious.

(d) Let $\mathbf{u}=\left(u_{1}, u_{2}, \ldots, u_{r}\right)$ be an optimum vector for $A$. Define $\mathbf{y}=$ $\left(y_{1}, y_{2}, \ldots, y_{r^{\prime}}\right)$ by $y_{i}=\sum\left\{u_{j}: f(j)=i\right\}\left(i=1,2, \ldots, r^{\prime}\right)$. Then $\sum y_{i}=1$ and, by (5.2),

$$
g\left(A^{\prime}\right) \geqslant \mathbf{y} A \mathbf{y}^{*}=\sum_{j=0}^{r} \sum_{k=0}^{r} \mathbf{a}_{f(j), f(k)}^{\prime} u_{j} u_{k} \geqslant \mathbf{u} A \mathbf{u}^{*}=g(A) .
$$

(e) For equality to hold in (5.3) all $y_{i}$ must be positive, so $f$ is onto; also, equality must hold in (5.2). Without limiting generality, suppose $f(1)=f(2)$. Defining $\mathbf{v}$ and $\mathbf{w}$ as in the proof of Lemma 4 , we see that $\mathbf{w} A \mathbf{w}^{*}=\mathbf{u} A \mathbf{u}^{*}$, contradicting the uniqueness of $\mathbf{u}$. Hence $f$ is also one-to-one. The permutation of rows and columns of $A$ given by $f$ transforms $(A, \mathbf{a})$ into $\left(A^{\prime}, \mathbf{a}\right)$.

REMARKS. (1) While it is obvious that $A \uparrow A^{\prime}$ if $A(\infty) \subseteq A^{\prime}(\infty)$, the converse is not true. For each positive integer $r$, let an $r \times r$ upper triangular matrix $A=\left(a_{i j}\right)$ be defined by

$$
a_{i j}= \begin{cases}2, & i<j \\ 0, & \text { otherwise }\end{cases}
$$

then $A \uparrow(1)$. In another example,

$$
A=\left[\begin{array}{llll}
0 & 2 & 0 & 2 \\
2 & 0 & 2 & 0 \\
2 & 2 & 0 & 2 \\
0 & 2 & 2 & 0
\end{array}\right], \quad A^{\prime}=\left[\begin{array}{lll}
1 & 2 & 2 \\
2 & 0 & 2 \\
2 & 2 & 0
\end{array}\right], \quad g(A)=\frac{7}{6}, \quad g\left(A^{\prime}\right)=\frac{3}{2}
$$

In both cases inclusion fails because of the type of order imposed on full classes.

(2) While the definition we have chosen for $A(\infty)$ appears a "natural" generalization, problems concerning order type are not central to our present study. We could also obtain the results we require by endowing its full classes with the order type $\omega^{*}+\omega$ of the integers, or $\lambda$, of the rationals.

The existence in a matrix digraph $A\langle\mathbf{x}\rangle$ of a subdigraph having "many" arcs induces a lower bound for the density $g(A)$ :

Lemma 6. Let $A \in \mathscr{D}$. Then $g(A)=\operatorname{Max}\left\{2\left(e\left(G^{n}\right) / n^{2}\right): G^{n}\right.$ is $A$-colourable $\}$.

Proof. If $G^{n} \subseteq A\langle\mathbf{x}\rangle$, where $\sum x_{i}=n$, then, by (3.2), $e\left(G^{n}\right) / n^{2} \leqslant e(A(n)) / n^{2}=$ $g(A)+O(1 / n)$ as $n \rightarrow \infty$. But $A(n)$ is $A$-colourable.

6. Augmentation of matrices. Our basic technique for proving digraph extremal theorems is to show that, if $G^{n}$ has "many" arcs, there must exist suitable dense matrices $A$ of sufficiently high density such that $G^{n}$ contains optimal matrix digraphs $A(m)$. Our reasoning will involve a recursive procedure we call augmentation, at each iteration of which a matrix is replaced by one of higher density. 
DEFINITION 10. (a) Let $r \geqslant 1,1 \leqslant k \leqslant r+1, \quad B=\left(a_{i j}\right)_{i, j=1, \ldots, r+1}, \quad \mathbf{b}=$ $\left(a_{1}, \ldots, a_{r+1}\right), \quad(B, \mathbf{b}) \in \mathscr{M}_{r+1}, \quad A=\left(a_{i j}\right)_{i, j=1, \ldots, k-1, k+1, \ldots, r+1}, \quad \mathbf{a}=\left(a_{1}, \ldots, a_{k-1}\right.$, $\left.a_{k+1}, \ldots, a_{r+1}\right),(A, \mathbf{a}) \in \mathscr{D}_{r}$; that is, $A$ is obtained by deleting the $k$ th row and column of $B$. Let $\mathbf{u}$ be the optimum vector of $A$. If

$$
\gamma=\frac{1}{2} \sum_{\substack{j=1 \\ j \neq k}}^{r+1}\left[a_{k, j}+a_{j, k}\right] u_{j}>g(A),
$$

we say that $B$ is obtained from $A$ by augmentation by $\gamma$, or that $B$ is an augmentation of $A$. We call any matrix $A^{\prime}=D(B)$ a reduced augmentation of $A$ by $\gamma$. We also regard any $1 \times 1$ matrix as an augmentation of $\varnothing$ by zero. Where $k-$ the number of the augmenting row and column-is not specified, it will be taken to be $r+1$.

(b) $a_{k k}$ and $a_{k}$ may have any values consistent with (2.1).

REMARKS. (1) Let $\mathbf{x}=\left(x_{1}, x_{2}, \ldots, x_{r}\right), \quad m=\sum_{i=1}^{r} x_{i}, \quad m^{\prime}=[1-g(A) / \gamma] m+$ $o(m), \mathbf{z}=\left(x_{1}, x_{2}, \ldots, x_{r}, m^{\prime}\right), n=m+m^{\prime}$. Motivation for the preceding definition comes from a recurrent situation wherein we shall wish to conclude, from the existence in a digraph $G^{n}$ of an optimal matrix subdigraph of structure $A\langle\mathbf{x}\rangle$ and from information of the form $e\left(G^{n}\right) \geqslant(g(A)+c)\left(\begin{array}{c}n \\ 2\end{array}\right)$, that $G^{n}$ contains optimal matrix digraphs $B\langle\mathbf{z}\rangle$, where $A$ is a proper principal submatrix of $B, g(B)>g(A)$. By (3.1),

$$
\begin{aligned}
e(B\langle\mathbf{z}\rangle) & \geqslant \frac{1}{2}\left[\mathbf{x} A \mathbf{x}^{*}+m^{\prime} \sum_{i=1}^{r}\left(a_{r+1, i}+a_{i, r+1}\right) x_{i}+O(m)\right] \\
& =\frac{1}{2}\left\{g(A) m^{2}+2 m m^{\prime} \gamma+O(m)+m m^{\prime} o(1)\right\} \text { as } m \rightarrow \infty
\end{aligned}
$$

( $\gamma$ defined by $(6.1)$ ). Hence

$$
\begin{aligned}
e(B(n)) & \geqslant\left(g(A)+\frac{\{\gamma-g(A)\}^{2}}{2 \gamma-g(A)}\right)\left(\begin{array}{l}
n \\
2
\end{array}\right)+o\left(n^{2}\right) \\
& =\frac{1}{2} \frac{\gamma^{2}}{2 \gamma-g(A)}\left(\begin{array}{l}
n \\
2
\end{array}\right)+o\left(n^{2}\right) \text { as } n \rightarrow \infty
\end{aligned}
$$

and $g(B)>g(A)$ (cf. (3.2)). (Usually $\mathbf{z}$ will not be the optimum vector for $B$.) To conclude this we require that there exist sufficiently many vertices $m^{\prime}$ outside of the optimal matrix digraph $A(m)$, all having the same types of adjacency to and from all vertices of each class of vertices of the $A(m)$.

(2) Augmentation "by at least $\gamma$ " or "by more than $\gamma$ " (briefly "by $\geqslant \gamma$ " or "by $>\gamma$ ") will mean augmentation by $\gamma^{\prime}$ such that $\gamma^{\prime} \geqslant \gamma$ or $\gamma^{\prime}>\gamma$, respectively.

(3) Wherc we wish to emphasize that a single application of the augmentation process is involved, without iteration, we may speak of direct augmentation.

LeMma 7. Let $A \in \mathscr{D}$.

(a) [3, LeMmA 2]. If $B$ is a (direct) augmentation of $A$ by $\gamma>g(A)$,

$$
g(B) \geqslant g(A)+\frac{\{\gamma-g(A)\}^{2}}{2 \gamma-g(A)}>g(A) .
$$


(b) Iterative augmentation [6, Lemma 8]. A sequence $\left\{A_{j}\right\}$ of dense matrices is defined recursively. Let $A_{0}=A$. If $g\left(A_{j}\right) \geqslant \gamma$, define $A_{j+1}=A_{j}$; if $g\left(A_{j}\right)<\gamma$, let $A_{j}^{\prime}$ be any augmentation of $A_{j}$ by at least $\gamma$, and choose $A_{j+1}=D\left(A_{j}^{\prime}\right)(j=0,1, \ldots)$. Then $\lim _{j \rightarrow \infty} g\left(A_{j}\right) \geqslant \gamma$. Moreover, there exists for every $\varepsilon>0$ an integer $K=$ $K(\gamma, \varepsilon)$, independent of $\left\{A_{j}\right\}$, such that $g\left(A_{j}\right)>\gamma-\varepsilon$ when $j>K$.

(c) [3, Lemma 4]. For any real numbers $\varepsilon>\varepsilon^{\prime}>0$, and positive integer $m$, there exists an integer $m_{0}=m_{0}\left(A, \varepsilon, \varepsilon^{\prime}, m\right)$ such that, for any sequence $\left\{G^{n}\right\}$, if (i) $G^{n}$ contains a subdigraph $A\left(m_{0}\right)$; (ii) every valency ${ }^{9}$ in $G^{n}$ exceeds $(g(A)+\varepsilon) n$; and (iii) $n$ is sufficiently large, there exists a reduced augmentation $A^{\prime}$ of $A$ by $\geqslant g(A)+\varepsilon^{\prime}$ for which $G^{n}$ contains $A^{\prime}(m)$.

(d) Let $A$ be weakly contained in a sequence $\left\{G^{n}\right\}$ of digraphs, respectively of minimum valency $\delta\left(G^{n}\right)$. Suppose that $\gamma=\liminf _{n \rightarrow \infty}\left(\delta\left(G^{n}\right) / n\right)>g(A)$. Then there exists a direct augmentation $A^{\prime}$ of $A$ by at least $\gamma$ which is weakly contained in $\left\{G^{n}\right\}$.

Proof of (d). Suppose that $0<2 \varepsilon<\gamma-g(A)$. For any integer $m$ and for infinitely many $n, G^{n}$ contains a subdigraph $A\left(m_{0}(A, \gamma-g(A)-\varepsilon, \gamma-g(A)-\right.$ $2 \varepsilon, m)$ ). For each such $n$ there exists an augmentation $A_{n, m}^{\prime}$ of $A$ by $\geqslant \gamma-2 \varepsilon$ such that $G^{n}$ contains $A_{n, m}^{\prime}(m)$. As $A$ admits only finitely many augmentations, we may conclude, by taking $\varepsilon$ sufficiently small, that there is one augmentation $A^{\prime}$ of $A$ by at least $\gamma$ such that $A^{\prime}(m) \subseteq G^{n}$ for infinitely many $n$. Hence there exists some $A^{\prime}$ which is weakly contained in $\left\{G^{n}\right\}$.

We shall require the following result for $q=1$, announced in [3, Theorem 3]. The proof will be given in the next section.

Theorem 5. Let $q-1, A=\left(a_{i j}\right) \in \mathscr{D}$. If $a_{k k}=1$, then $a_{j k}=a_{k j}=2(j \neq k)$.

Remarks. (1) The following converse of Theorem 5 holds for all $q$ (cf. $\$ 3$, Example 1): Let $A$ and $B$ be square matrices, and $J$ any suitable square matrix of 1 's. Then the matrix

$$
\left(\begin{array}{c|c}
A & 2 q J \\
\hline 2 q J & B
\end{array}\right)
$$

is dense if and only if both $A$ and $B$ are dense.

(2) As a first step towards proving Theorem 5, we have

Lemma 8 [3, Lemma 3]. Let $q=1$. Suppose that $B \in \mathscr{M}_{r+1}$ is an augmentation of $A=\left(a_{i j}\right)_{i, j=1,2, \ldots, r} \in \mathscr{D}_{r}$ such that, for some $i(1 \leqslant i \leqslant r)$,

$$
a_{i j}=a_{j i}= \begin{cases}1, & j=i, \\ 2, & 1 \leqslant j \leqslant r, j \neq i .\end{cases}
$$

Then $a_{r+1, i}=a_{i, r+1}=2$, and every $D(B)$ contains entries from the ith row and column of $B$.

\footnotetext{
${ }^{9}$ In-valency + out-valency.
} 
Proof. Let $\mathbf{u}=\left(u_{1}, u_{2}, \ldots, u_{r}\right)$ be the optimum vector of $A$. By (6.1) and (5.1),

hence

$$
4 \geqslant \sum_{j=1}^{r}\left(a_{r+1, j}+a_{j, r+1}\right) u_{j}>2 g(A)=\sum_{j=1}^{r}\left(a_{i j}+a_{j i}\right) u_{j}=4-2 u_{i} \text {; }
$$

$$
2 u_{i}>\sum_{j=1}^{r}\left(4-a_{r+1, j}-a_{j, r+1}\right) u_{j} \geqslant\left(4-a_{r+1, i}-a_{i, r+1}\right) u_{i} \geqslant 0,
$$

and $a_{r+1, i}=a_{i, r+1}=2$. If $D(B)$ contained no entries from the $i$ th row of $B$, we could augment $D(B)$ by 2 to obtain a principal submatrix of $B$ whose density exceeds $g(D(B))$.

EXAmple. If $A^{\prime}$ is a reduced augmentation of $A$, it need not follow that $A \uparrow A^{\prime}$. Let $q=1$,

$$
A=\left(\begin{array}{llll}
0 & 2 & 0 & 2 \\
2 & 0 & 2 & 0 \\
2 & 2 & 0 & 2 \\
0 & 2 & 2 & 0
\end{array}\right), \quad A^{\prime \prime}=\left(\begin{array}{llll|l}
0 & 2 & 0 & 2 & 0 \\
2 & 0 & 2 & 0 & 2 \\
2 & 2 & 0 & 2 & 2 \\
0 & 2 & 2 & 0 & 0 \\
\hline 0 & 2 & 2 & 0 & 0
\end{array}\right), \quad A^{\prime}=\left(\begin{array}{lll}
0 & 2 & 2 \\
2 & 0 & 2 \\
2 & 2 & 0
\end{array}\right)
$$

$A^{\prime \prime}$, having density $\frac{4}{3}$, is an augmentation of $A$, a dense matrix with optimum vector $\mathbf{u}=\left(\frac{1}{6}, \frac{1}{3}, \frac{1}{3}, \frac{1}{6}\right)$ and density $g(A)=\frac{7}{6} . A^{\prime}=D\left(A^{\prime \prime}\right)$ is obtained by deleting the 1 st and 4 th rows and columns of $A$. For $m \geqslant 4, A^{\prime}(\infty)$ cannot contain $A(m)$, since $\chi(A(m)) \geqslant 4$.

\section{Augmentation schemes.}

Motivation. We can approach the question of whether or not digraphs in a sequence $\left\{G^{n}\right\}$ contain an $L \in \mathscr{L}$ by seeking a matrix $A$ such that some $A(m)$ can be interposed between some $L \in \mathscr{L}$ and $G^{n}$; determination of $A$ usually involves iterative augmentations. We may also proceed systematically: Beginning with $\varnothing$, we determine iterated augmentations contained weakly in $\left\{G^{n}\right\}$. Any matrix $A$, each of whose augmentations $A^{\prime}$ is prohibited by $\mathscr{L}$ (in the sense that some $L \in \mathscr{L}$ is $A^{\prime}$-colourable), is identified as an end-matrix, and augmented no further. Should the procedure terminate, we have a "finite augmentation scheme". Defining $\gamma$ ' to be the maximum density of matrices in the scheme, we argue that, for any $\varepsilon>0$ and for $n$ sufficiently large, any graph $G^{n}$ of the sequence with $e\left(G^{n}\right) \geqslant\left(\gamma^{\prime} / 2+\varepsilon\right) n^{2}$ must contain $L \in \mathscr{L}$. These schemes are specified by three types of information: (i) by the rate at which the densities are permitted to increase-more precisely, by a lower bound on $\gamma$ in (6.1); (ii) by the rule by which a matrix is designated an end-matrix; (iii) by restrictions on the population of matrices from which an augmentation may be considered.

DEFINITION 11. Let $\left\{\mathscr{S}_{i}\right\}_{i=0,1, \ldots}$ be a sequence of disjoint sets of dense matrices; let $\mathscr{T}_{n}=\bigcup_{i=0}^{n} \mathscr{S}_{i}, \mathscr{T}=\bigcup_{n=0}^{\infty} \mathscr{S}_{n}$. A directed graph $\mathscr{A}$ with vertex set $\mathscr{T}$ is an augmentation scheme if

(a) an arc is directed from $A$ to $A^{\prime}$ only if $A^{\prime}$ is a reduced augmentation of $A$;

(b) directed to every matrix in $\mathscr{S}_{i+1}$ there is at least one arc from a vertex in $\mathscr{S}_{i}$ $(i \geqslant 0)$;

(c) all arcs emanating from matrices in $\mathscr{S}_{i}$ terminate in $\mathscr{T}_{i+1}(i \geqslant 0)$.

Vertices of $\mathscr{A}$ whose out-valency is 0 will be called end-vertices. 
Definition 12. (a) Let $\gamma$ be a real number, $\mathscr{L}$ a (possibly empty) family of digraphs, and $\mathscr{S}_{0}$ a nonempty set of dense matrices each weakly contained in a sequence $\left\{G^{n}\right\}$ of digraphs. The augmentation scheme $\mathscr{A}\left(\mathscr{S}_{0}, \gamma,\left\{G^{n}\right\}\right)$ is defined recursively as follows: $\mathscr{S}_{n}$ having been defined, define $\mathscr{U}_{n}$ to be the set of reduced augmentations $A^{\prime}$ of any $A \in \mathscr{S}_{n}$ such that

$$
A^{\prime} \text { is a reduced augmentation of } A \text { by } \geqslant \gamma,
$$

$$
g(A)<\gamma,
$$

$A^{\prime}$ is weakly contained in $\left\{G^{n}\right\}$,

and let $\mathscr{S}_{n+1}=\mathscr{U}_{n} \backslash \bigcup_{i=0}^{n} \mathscr{S}_{i}$. The vertex set of the scheme is $\bigcup_{n=0}^{\infty} \mathscr{S}_{n}$; where conditions (7.1)-(7.3) are satisfied, an arc is directed from $A$ to $A^{\prime}$. The scheme $\mathscr{A}^{+}\left(\mathscr{S}_{0}, \gamma,\left\{G^{n}\right\}\right)$ is defined analogously, replacing (7.1), (7.2) by

$$
A^{\prime} \text { is a reduced augmentation of } A \text { by }>\gamma,
$$

$$
g(A) \leqslant \gamma .
$$

We speak of augmentation in $\left\{G^{n}\right\}$. When $G^{n}=B(n), B \in \mathscr{D}$, we speak of augmentation in $B$, and, may write $\mathscr{A}\left(\mathscr{S}_{0}, \gamma, B\right)$ or $\mathscr{A}^{+}\left(\mathscr{S}_{0}, \gamma, B\right)$.

(b) When condition (7.3) is superfluous, we may abbreviate the notation to $\mathscr{A}\left(\mathscr{S}_{0}, \gamma\right)$ and $\mathscr{A}^{+}\left(\mathscr{S}_{0}, \gamma\right)$.

(c) When conditions (7.1) and (7.2) are replaced by

$$
A^{\prime} \text { is a reduced augmentation of } A \text {, }
$$

we obtain the complete augmentation scheme $\mathscr{A}\left(\mathscr{S}_{0}\right)$.

(d) Let $\mathscr{L}$ be a fixed family of digraphs. If we adjoin to conditions (7.1)-(7.3) the additional requirement

$$
\text { no } L \in \mathscr{L} \text { is } A^{\prime} \text {-colourable }
$$

we obtain an augmentation scheme $\mathscr{A}\left(\mathscr{S}_{0}, \gamma,\left\{G^{n}\right\} \bmod \mathscr{L}\right)$; where condition (7.3) is superfluous we write $\mathscr{A}\left(\mathscr{S}_{0}, \gamma \bmod \mathscr{L}\right)$. We speak of augmentation modulo $\mathscr{L}$.

REMARKs. (1) Lemma 7(b) is concerned with a scheme $\mathscr{A}\left(\left\{A_{0}\right\}\right)$.

(2) In the proof of Theorem 4 we shall exploit a subscheme $\mathscr{A}(\{\varnothing\}, \gamma \bmod \mathscr{L})$, wherein augmentations at the $j$ th level will be "by" a constant $\gamma_{j}$ determined by the matrices in levels 0 through $j$.

Lemma 9. Let $A \in \mathscr{D}_{r}, \bar{A} \in \mathscr{D}_{\dot{r}} . \varnothing \neq A \uparrow \bar{A}$.

(a) If $A \neq \bar{A}$, there exists a direct augmentation $A^{\prime}$ of $A$ by $\geqslant g(\bar{A})$ such that $A^{\prime} \uparrow \bar{A}$.

(b) There exists a finite sequence $A=A_{0}, A_{1}, \ldots, A_{p}=\bar{A}$ such that $A_{i} \uparrow \bar{A}$ and $A_{i}$ is a reduced augmentation of $A_{i-1}$ by at least $g(\bar{A})(i=1,2, \ldots, p)$.

(c) $\bar{A}$ is a direct augmentation of one of its proper submatrices.

(d) The set of matrices in $\mathscr{A}(\{\varnothing\})$ can be generated by recursive augmentations of $\varnothing$ without reduction; any dense $r \times r$ matrix can be reached through a chain of precisely $r$ dense matrices, each augmenting its predecessor, beginning with $\varnothing$. 
Proof. (a) By Lemma 5(d) and (e), $g(A)<g(\bar{A})$. Apply Lemmas 3(b), 7 to $\left\{G^{n}\right\}=\{\bar{A}(n)\}$.

(b) Suppose that $f^{\prime}: A^{\prime} \uparrow \bar{A}$ is the canonical embedding. If any entries in the augmenting $(r+1)$ st row and column of $A^{\prime}$ are not equal to the corresponding values in $\bar{A}$, replace the offending values in $A^{\prime}$ by the larger entries from $\bar{A}$, thereby obtaining another matrix which surely augments $A$ by at least $g(\bar{A}) . f^{\prime}(r+1) \neq$ $f^{\prime}(i)$ for all $i \neq r$. We apply (a) to $A_{1}=A^{\prime}$, to obtain, after reduction, $A_{2}$. The process is repeated, and must terminate after finitely many applications, since the number of rows and columns of $A_{i}$ will never exceed $r+\bar{r}$.

(c) Among all dense proper principal submatrices of $\bar{A}$, let $\tilde{A}$ denote one whose density is maximal. By (a), $\tilde{A}$ admits a direct augmentation $A^{\prime}$ having a canonical embedding $f: A^{\prime} \uparrow \bar{A}$. The maximality assumption ensures that $f$ is one-to-one and onto. $\bar{A}$ must be a direct augmentation of $\tilde{A}$.

REMARKS. (1) While Lemma 9(b) asserts the existence of a finite chain, there can exist infinite chains. For example, if $A_{i}$ is the $i \times i$ triangular matrix with zeros below and on the main diagonal, and 2's above (i.e. twice the adjacency matrix of a suitably labelled transitive tournament on $i$ vertices), then $A_{i}$ is an augmentation of $A_{i-1}$ by $\geqslant 1, A_{i} \uparrow \bar{A}(1)(i=1,2, \ldots)$.

(2) Lemma 9(b) implies that the restriction of $\uparrow$ to dense matrices is contained in the transitive closure of the reduced augmentation relation.

(3) Proof of Theorem 5. By Lemma 9(b) there exists a finite sequence $(1)=$ $A_{0}, A_{1}, \ldots, A_{p}=A$ such that $A_{i}$ is a reduced augmentation of $A_{i-1}(i=1,2, \ldots, p)$. Repeated applications of Lemma 8 imply that any row or column of $A$ with diagonal entry 1 has all other entries equal to 2 .

Lemma 10 (Approximation Lemma). Let $\mathscr{S}_{0} \subset \mathscr{D}, \varepsilon>0$, and let $\left\{G^{n}\right\}$ be $a$ sequence of digraphs such that

$$
\liminf _{n \rightarrow \infty} \frac{\delta\left(G^{n}\right)}{n} \geqslant \gamma
$$

Then any nonempty augmentation scheme $\mathscr{A}\left(\mathscr{S}_{0}, \gamma,\left\{G^{n}\right\}\right)$ must contain a matrix $A$ such that $g(A)>\gamma-\varepsilon$.

Proof. Let $A_{0}$ be a matrix in the scheme such that $g\left(A_{0}\right) \leqslant \gamma-\varepsilon$. By (7.8), for sufficiently large $n, \delta\left(G^{n}\right) \geqslant(\gamma-\varepsilon / 2) n \geqslant\left\{g\left(A_{0}\right)+\varepsilon / 2\right\} n$. Lemma 7 ensures the existence of a reduced augmentation of $A_{0}$ by $\geqslant \gamma$ which is also weakly contained in $\left\{G^{n}\right\}$. We obtain, after at most $K(\gamma-\varepsilon / 2, \varepsilon / 2)$ iterations, a matrix in the scheme, weakly contained in $\left\{G^{n}\right\}$, and having density exceeding $\gamma-\varepsilon$.

The preceding lemma may be applied to prove the following weakened version of Theorem A, valid for all $q$.

LEMMA 11. Let $\mathscr{L}$ be a family of digraphs.

(a) [7, Lemma 2]. The sequence $\left\{\operatorname{ex}(n, \mathscr{L}) /\left(\begin{array}{c}n \\ 2\end{array}\right)\right\}$ decreases monotonically.

(b) Let $\gamma=\lim _{n \rightarrow \infty}\left\{\operatorname{ex}(n, \mathscr{L}) / n^{2}\right\}$. Let $\varepsilon>0$. Then the augmentation scheme $\mathscr{A}(\{\varnothing\}, \gamma \bmod \mathscr{L})$ contains a matrix $A$ such that $\gamma-\varepsilon<g(A) \leqslant \gamma$.

(c) Let $\alpha>\gamma=\lim _{n \rightarrow \infty}\left\{\operatorname{ex}(n, \mathscr{L}) / n^{2}\right\}$. Then any augmentation scheme $\mathscr{A}(\{\varnothing\}$, $\alpha \bmod \mathscr{L})$ is finite. 
Proof. (b) Let $S^{n} \in \operatorname{EX}(n, \mathscr{L})(n=1,2, \ldots)$. By (a), $e\left(S^{n}\right)=\gamma n^{2} / 2+o\left(n^{2}\right)$ as $n \rightarrow \infty$. A standard technique [3, p. 91] yields a sequence of subdigraphs $U^{m(n)} \subseteq S^{n}$ for which $m(n)$ is strictly increasing and $\delta\left(U^{m}\right) \geqslant(\gamma-\varepsilon / 2) m$ for $m$ sufficiently large. By Lemma 10 there exists in the scheme $\mathscr{A}\left(\{\varnothing\}, \gamma,\left\{U^{m(n)}\right\}\right)$ a matrix $A$ such that $g(A)>\gamma-\varepsilon$, and that no $L \in \mathscr{L}$ is $A$-colourable.

(c) By Lemma 7, sufficiently many iterated augmentations yield a matrix $A$ of density exceeding $\gamma$. By (3.2), $e(A(n))>\operatorname{ex}(n, \mathscr{L})$ for $n$ sufficiently large, so some $L \in \mathscr{L}$ is $A$-colourable.

EXAMPLE. For $q=1$ we may determine the first levels of the complete augmentation scheme $\mathscr{A}(\varnothing)$ recursively. We tabulate (up to equivalence) the matrices in the first four levels, and their optimum vectors.

\begin{tabular}{|c|c|c|c|}
\hline Level & Matrix & Optimum Vector & Density \\
\hline 0 & $\varnothing$ & & $-\infty$ \\
\hline \multirow[t]{2}{*}{1} & (0) & (1) & 0 \\
\hline & (1) & (1) & 1 \\
\hline \multirow[t]{3}{*}{2} & $\left(\begin{array}{ll}0 & 2 \\
0 & 0\end{array}\right)$ & $\left(\frac{1}{2}, \frac{1}{2}\right)$ & $\frac{1}{2}$ \\
\hline & $\left(\begin{array}{ll}0 & 2 \\
2 & 0\end{array}\right)$ & $\left(\frac{1}{2}, \frac{1}{2}\right)$ & 1 \\
\hline & $\left(\begin{array}{ll}0 & 2 \\
2 & 1\end{array}\right)$ & $\left(\frac{1}{3}, \frac{2}{3}\right)$ & $\frac{4}{3}$ \\
\hline \multirow[t]{8}{*}{3} & $\left(\begin{array}{lll}0 & 2 & 2 \\
0 & 0 & 2 \\
0 & 0 & 0\end{array}\right)$ & $\left(\frac{1}{3}, \frac{1}{3}, \frac{1}{3}\right)$ & $\frac{2}{3}$ \\
\hline & $\left(\begin{array}{lll}0 & 2 & 0 \\
0 & 0 & 2 \\
2 & 0 & 0\end{array}\right)$ & $\left(\frac{1}{3}, \frac{1}{3}, \frac{1}{3}\right)$ & $\frac{2}{3}$ \\
\hline & $\left(\begin{array}{lll}0 & 2 & 2 \\
2 & 0 & 2 \\
2 & 0 & 0\end{array}\right)$ & $\left(\frac{3}{7}, \frac{2}{7}, \frac{2}{7}\right)$ & $\frac{8}{7}$ \\
\hline & $\left(\begin{array}{lll}0 & 2 & 2 \\
2 & 0 & 2 \\
2 & 2 & 0\end{array}\right)$ & $\left(\frac{1}{3}, \frac{1}{3}, \frac{1}{3}\right)$ & $\frac{4}{3}$ \\
\hline & $\left(\begin{array}{lll}1 & 2 & 2 \\
2 & 0 & 2 \\
2 & 0 & 0\end{array}\right)$ & $\left(\frac{3}{5}, \frac{1}{5}, \frac{1}{5}\right)$ & $\frac{7}{5}$ \\
\hline & $\left(\begin{array}{ll}1 & 2 \\
2 & 1\end{array}\right)$ & $\left(\frac{1}{2}, \frac{1}{2}\right)$ & $\frac{3}{2}$ \\
\hline & $\left(\begin{array}{lll}1 & 2 & 2 \\
2 & 0 & 2 \\
2 & 2 & 0\end{array}\right)$ & $\left(\frac{1}{2}, \frac{1}{4}, \frac{1}{4}\right)$ & $\frac{3}{2}$ \\
\hline & $\left(\begin{array}{lll}1 & 2 & 2 \\
2 & 1 & 2 \\
2 & 2 & 0\end{array}\right)$ & $\left(\frac{2}{5}, \frac{2}{5}, \frac{1}{5}\right)$ & $\frac{8}{5}$ \\
\hline
\end{tabular}


Suppose $A \in \mathscr{D}, 1<g(A)<\frac{8}{7}$. By Lemma 9 and the preceding computations we know that $A$ can only be a (possibly iterated) reduced augmentation of

$$
\left(\begin{array}{lll}
0 & 2 & 2 \\
0 & 0 & 2 \\
0 & 0 & 0
\end{array}\right) \text { or }\left(\begin{array}{lll}
0 & 2 & 0 \\
0 & 0 & 2 \\
2 & 0 & 0
\end{array}\right) \text {. }
$$

We may prove by induction that no such $A$ exists.

8. "Splitting" a dense matrix. We pause to forge a technical tool needed in our proof of the Main Lemma. The concept of "splitting" will permit approximation of a dense matrix by one of almost the same density, having zeros on its main diagonal.

Definition 13. Let $(A, \mathbf{a}) \in \mathscr{D}_{r}$, and let $k$ be an integer, $1 \leqslant k \leqslant r$.

(a) $\left(A^{\prime}, \mathbf{a}^{\prime}\right) \in \mathscr{M}_{r+1}$ is a stretching of the $k$ th row and column of $(A, \mathbf{a})$ if

$$
\begin{gathered}
a_{i j}^{\prime}=a_{i j}, \quad 1 \leqslant i, j \leqslant r ;(i, j) \neq(k, k) ; \\
a_{r+1, j}^{\prime}=a_{k, j}, \quad a_{j, r+1}^{\prime}=a_{j, k}, \quad a_{j}^{\prime}=a_{j}, \quad 1 \leqslant j \leqslant r ; j \neq k ; \\
a_{k, r+1}^{\prime}=2 a_{k}, \quad a_{r+1, k}^{\prime}=2\left(a_{k k}-a_{k}\right) ; \\
a_{k, k}^{\prime}=a_{r+1, r+1}^{\prime}=a_{k, k}, \quad a_{k}^{\prime}=a_{r+1}^{\prime}=a_{k} .
\end{gathered}
$$

(b) $\left(A^{\prime}, \mathbf{a}^{\prime}\right) \in \mathscr{M}_{r+1}$ is a splitting of the $k$ th row and column of $(A, \mathbf{a})$ if $(8.1),(8.2)$ hold, and also

$$
\begin{gathered}
a_{k, r+1}^{\prime}=2 a_{k}+2, \quad a_{r+1, k}^{\prime}=2\left(a_{k k}-a_{k}\right), \\
a_{k, k}^{\prime}=a_{r+1, r+1}^{\prime}=a_{k, k}-1 \geqslant 0, \quad a_{k}^{\prime}=a_{r+1}^{\prime}=\operatorname{Max}\left\{a_{k}-1,0\right\} .
\end{gathered}
$$

LemMa 12. Let $(A, \mathbf{a}) \in \mathscr{D}_{r}$.

(a) Let $\left(A^{\prime}, \mathbf{a}^{\prime}\right)$ be a stretching of $(A, \mathbf{a})$. Then $\left(A\right.$, a) is not dense, but $\left(A^{\prime}, \mathbf{a}^{\prime}\right) \uparrow(A, \mathbf{a})$ and $g\left(A^{\prime}\right)=g(A)$.

(b) Let $\left(A^{\prime}, \mathbf{a}^{\prime}\right)$ be a splitting of $(A, \mathbf{a})$. Then $\left(A\right.$, a) is dense, and $g\left(A^{\prime}\right)=g(A)$.

(c) There exists $\left(A^{\prime}, \mathbf{a}^{\prime}\right) \in \mathscr{D}$ having zero trace, and such that $g\left(A^{\prime}\right)=g(A)$.

Proof. (a) Evidently

$$
\begin{aligned}
& \left(x_{1}, x_{2}, \ldots, x_{k}, \ldots, x_{r}, x_{r+1}\right) A^{\prime}\left(x_{1}, x_{2}, \ldots, x_{k}, \ldots, x_{r}, x_{r+1}\right)^{*} \\
& \quad=\left(x_{1}, x_{2}, \ldots, x_{k}+x_{r+1}, \ldots, x_{r}\right) A\left(x_{1}, x_{2}, \ldots, x_{k}+x_{r+1}, \ldots, x_{k}\right)^{*},
\end{aligned}
$$

so $g\left(A^{\prime}\right) \leqslant g(A)$. But $A$ is a proper submatrix of $A^{\prime}$, so $g(A) \leqslant g\left(A^{\prime}\right)$.

(b)

$$
\begin{aligned}
\left(x_{1}, x_{2}, \ldots, x_{k}, \ldots, x_{r}, x_{r+1}\right) A^{\prime}\left(x_{1}, x_{2}, \ldots, x_{k}, \ldots, x_{r}, x_{r+1}\right)^{*} & \\
= & \left(x_{1}, x_{2}, \ldots, x_{k}+x_{r+1}, \ldots, x_{r}\right) A^{\prime}\left(x_{1}, x_{2}, \ldots, x_{k}+x_{r+1}, \ldots, x_{r}\right)^{*} \\
& -\left(x_{k}-x_{r+1}\right)^{2} .
\end{aligned}
$$

Hence $g\left(A^{\prime}\right) \leqslant g(A)$. If $\mathbf{u}=\left(u_{1}, u_{2}, \ldots, u_{k}, \ldots, u_{r}\right)$ is the optimum vector of $A$, and $\mathbf{v}=\left(u_{1}, u_{2}, \ldots, u_{k-1}, u_{k} / 2, u_{k+1}, \ldots, u_{r}, u_{k} / 2\right)$, then $\mathbf{v} A^{\prime} \mathbf{v}^{*}=g(A)$, so $g\left(A^{\prime}\right)=$ $g(A)$. It follows that $A^{\prime}$ is dense.

(c) Iterations of the splitting operation progressively reduce the sum $\sum_{i} 3^{a_{i i}}$. 
It is possible to "approximate" a dense matrix by matrices with zeros in the main diagonal, using the operation of stretching followed by suppression of the new diagonal nonzero elements. More precisely, we have the following

Lemma 13. For any $(A, \mathbf{a}) \in \mathscr{D}_{r}, \varepsilon>0$, there exists $\left(A^{\prime}, \mathbf{a}^{\prime}\right) \in \mathscr{D}$ such that $\left(A^{\prime}, \mathbf{a}^{\prime}\right) \uparrow(A, \mathbf{a})$ and $g\left(A^{\prime}\right)>g(A)-\varepsilon$, where all main diagonal entries in $A^{\prime}$ are zero.

Proof. It suffices to prove the case where $A$ has exactly one nonzero main diagonal element, which we take to be in position (1.1). In graph-theoretical terms, the operation described below corresponds to partitioning the first class of $A(n)$ into many parts, and suppressing arcs having both ends within any one part. Let $k$ $=\lceil q / \varepsilon\rceil$. After $k$ iterated stretchings of the first row and column, and rearrangement, $A$ becomes a matrix of the form

$$
A_{k}=\left(\begin{array}{c|c}
U & X \\
\hline Y & A
\end{array}\right)
$$

where $U$ has all main diagonal entries equal to $a_{11}$, all entries below the diagonal equal $2 a_{i}$, and all above the diagonal equal $2 a_{i i}-2 a_{i} ; X$ consists of $k$ identical rows which coincide with the first row of $A$, but with the first entry of each replaced by $2 a_{i}$; analogously $Y$. Then let the first $k+1$ main diagonal entries of $A_{k}$ be replaced by zeros, to form a new matrix we call $A_{k}^{\prime}$; evidently $A_{k}^{\prime} \uparrow A, g(A) \geqslant g\left(A_{k}^{\prime}\right)$ $\geqslant g(A)-\varepsilon$. Take $A^{\prime}=D\left(A_{k}^{\prime}\right)$.

9. The Main Lemma. Proofs of Theorems 1-4 will involve application of the following

Lemma 14 (Main Lemma). Let $q=1, A \in \mathscr{D}$. There exists a constant $c=c_{A}$ and $a$ positive integer $m_{A}$ such that, for any $B \in \mathscr{D}$ satisfying

$$
g(A) \leqslant g(B)<g(A)+c, \quad B \approx A,
$$

no $A\left(m_{A}\right)$ is B-colourable.

We reformulate the lemma more explicitly.

Lemma $14^{\prime}$. Let $q=1, A \in \mathscr{D}_{r}$. Define $c=c_{A}=\frac{1}{2} \operatorname{Min}\left\{g\left(A^{\prime}\right)-g(A)\right\}$ as $A^{\prime}$ ranges over all augmentations of $A$ and of matrices obtainable from $A$ by successive splittings. Then there exists an integer $m_{A}$ such that, for any $B \in \mathscr{D}$ satisfying (9.1) and for $n \geqslant m_{A}$, no $A(n)$ is B-colourable.

A digraph $A(m)$ may be embedded in $B(\infty)$ in such a way that the vertices of a full class of $A(m)$ are distributed one each over distinct independent classes of $B(\infty)$. As such an embedding is not canonical, we cannot apply Lemma 9. We cope with this situation by proving the existence of a large subdigraph-where each vertex of $A(m)$ is replaced by a set of vertices. More specifically, we shall apply the following construction.

Definition 14. Let $h$ be a positive integer, and $G$ a given digraph. By $G \circ I_{h}$ we shall mean the digraph obtained from $G$ by replacing each vertex $x$ by a class $\mathscr{I}_{x}$ of $h$ vertices, joining new vertices by arcs of the same multiplicity as those joining the vertices they replace. In other words, if $A$ is twice the adjacency matrix of $G$, then $G \circ I_{h}$ is defined to be $A\langle h, h, \ldots, h\rangle=A\langle h \mathbf{e}\rangle$. 
Proof of Lemma 14': (A) Outline. Our proof will be by induction on $s(A)$, defined to be the number of nonzero main diagonal entries in $A$. The case where $A$ has zero trace is disposed of in paragraph (C). At the critical step (paragraph (I)) we show-on the assumption that, for large $m$ and very large $n, A(m)$ is embedded in $B(n)$ - that there exists either an augmentation $A^{\prime}$ of $A$ such that $A^{\prime}(m) \subseteq B(n)$, or a splitting $A_{1}$ of $A$ such that $A_{1}(m) \subseteq B(n)$ : the former would contradict the choice of $c$, while the latter would violate the induction hypothesis.

(B) "Small" matrices $B$. Let $B \in \mathscr{D}_{\rho}$ be given, satisfying (9.1). There exists an integer $m_{1}=m_{1}(B)$ such that no $A\left(m_{1}\right)$ is $B$-colourable. (Otherwise $A \uparrow B$; by Lemma 9 there would exist a reduced augmentation $A^{\prime}$ of $A$ such that $A^{\prime} \uparrow B$; implying that $g(A)+2 c \leqslant g\left(A^{\prime}\right) \leqslant g(A)+c$.) Thus, if we consider first only those $B$ for which $\rho \leqslant m_{2}(A)$ - to be defined in $(\mathrm{F})$ - any value greater than the maximum of the values $m_{1}(B)$ will do for $m_{A}$. The main difficulty will be in coping with infinitely many $B$ 's simultaneously. We must prove the existence of $m_{A}$, independent of $B$, such that $A\left(m_{A}\right)$ is not $B$-colourable.

(C) Zero main diagonal. Let $s=0$. By Lemma 3 there exists an integer $m_{3}=m_{3}(A)$ such that, for every integer $n \geqslant m_{3}$, every class of every $A(n)$ is nonempty. As $s=0$, the $B$-colourability of $A\langle e\rangle$ would imply that of $A\left(m_{1}(B)\right)$. Hence $A\langle\mathbf{e}\rangle$ is not $B$-colourable, and we may take $m_{A}=m_{3}$ in this case.

(D) Difficulties. If $s \neq 0$, we must cope with two types of complications.

$\left(\mathrm{D}_{1}\right)$ It may happen that some $A(m)$ is $B$-colourable, while some $A\left(m^{\prime}\right)\left(m^{\prime}>m\right)$ is not: the vertices of some full class of the $A(m)$ could each be placed in a different class of $B(\infty)$.

$\left(\mathrm{D}_{2}\right) g(A)=\lim _{n \rightarrow \infty}\left(e(A(n)) /\left(\begin{array}{c}n \\ 2\end{array}\right)\right)$, a measure of the fraction of the total number of potential arcs realizable in a matrix digraph. When $A$ has zero main diagonal, and $m$ has suitable divisibility properties, this limit coincides with $\gamma_{m}=$ $\lim _{h \rightarrow \infty}\left(e\left(A(m) \circ I_{h}\right) /\left(\begin{array}{c}m h \\ 2\end{array}\right)\right)$. When $s>0, \gamma_{m}<g(A)$.

(E) Assume the lemma known for $s=s(A)-1$. Let $A, B$ satisfy (9.1). By Theorem 5 and Lemma 12, splitting any row and column of $A$ containing a nonzero diagonal entry always yields (up to equivalence) the same dense matrix $A_{1}$ for which $s\left(A_{1}\right)=s(A)-1, g(A)=g\left(A_{1}\right)$. Iterated splittings of $A_{1}$ are also iterated splittings of $A$, so $c_{A_{1}} \geqslant c_{A}$. Restrictions on $m_{2}$ below will imply that $m_{2}>r$. Hence $B \neq A_{1}$ and, by the induction hypothesis, there exists an integer $m_{4}=m_{4}(A)=m_{A_{1}}$ (independent of $B$ ) such that no $A_{1}\left(m_{4}\right)$ is $B$-colourable.

(F) By (3.2), there exists an integer $m_{5}\left(m_{5}>m_{4}\right)$ such that for all (of the finitely many) reduced augmentations $A^{\prime}$ of $A$, and for $m \geqslant m_{5}$,

$$
e\left(A^{\prime}(m)\right)>\frac{1}{2}\{g(A)+c\} m^{2} .
$$

Let $m_{6}=m_{6}(A)$ be the least common multiple of the denominators of the (rational) optimum vector $\mathbf{u}$ of $A$; let $m_{2}=m_{2}(A)=3 m_{5} m_{6}$. For any $s$ and $\rho \leqslant m_{2}(A)$, it suffices to take $m_{A}=m_{7}=\operatorname{Max}\left\{m_{1}(B): B\right.$ dense, $g(A) \leqslant g(B)<g(A)+c_{A}$, $\left.B \approx A, \rho \leqslant m_{2}(A)\right\}$.

(G) Let $\rho>m_{2}$. By Lemma 3 there exists an integer $m_{8}=m_{8}(A)>m_{2}+m_{3}$ such that, for $n \geqslant m_{8}$, every $A(n)$ contains $A\left\langle m_{2} \mathbf{u}\right\rangle$; let one such digraph be called $A\left[m_{2}\right]$, having $A$-classes $C_{1}, C_{2}, \ldots, C_{r}$. We assume that $A\left[m_{2}\right]$ is $B$-colourable, and 
derive a contradiction. $m_{A}$ will be taken to be the maximum of $m_{7}$ (for $\rho \leqslant m_{2}$ ) and $m_{8}$ (for $\rho>m_{2}$ ), both defined in terms of $A$. Let $\mathbf{v}$ be the optimum vector of $B$; henceforth we restrict $n$ to be divisible by the product of $m_{2}$ ! and the least common multiple of the reduced denominators of the (rational) coordinates of $\mathbf{v}$. Let $H^{n}$ be a digraph of structure $B\langle n \mathbf{v}\rangle$, having $B$-classes $\bar{C}_{1}, \bar{C}_{2}, \ldots, \bar{C}_{\rho}$. For $n$ sufficiently large, any embedding of $A\left[m_{2}\right]$ into $B(\infty)$ induces one into $H^{n}$. Among all such embeddings we fix one for which the number of classes of $H^{n}$ containing vertices of $A\left[m_{2}\right]$ is minimized. For this embedding all vertices of $C_{i}$ are contained together in one class of $H^{n}$, unless possibly $C_{i}$ is full and its vertices are each contained in a distinct independent class of $H^{n}$, containing no vertex of $C_{j}(i, j=1,2, \ldots, r ; i \neq j)$; and then every class of $H^{n}$ which does not contain embedded vertices of $A\left[m_{2}\right]$ is an independent class. (We have applied Theorem 5.)

(H) We now broaden our view to include the induced imbeddings into $H^{n}$ of digraphs $G_{1}^{m_{2} h}$ of structure $A\left[m_{2}\right] \circ I_{h}$, where each vertex $x$ of $A\left[m_{2}\right]$ is replaced by a set $\mathscr{I}_{x}$ of $h$ vertices, all in the same class $\bar{C}_{i}$ of $H^{n}$ containing $x$. More precisely, we require one such embedding that, for maximum $h$, completely exhausts the vertices in at least one $\bar{C}_{\vec{i}}$; this requirement is feasible since the number of vertices of $A\left[m_{2}\right]$ in any $\bar{C}_{i}$ divides $n v_{i}$. Let $G=G^{m_{2} h}$ be the subdigraph of $H^{n}$ spanned by $G_{1}^{m_{2} h}$, and let $e_{G}$ denote the number of arcs joining vertices of $G$ to vertices of $H^{n}-G$. Without limiting generality, we assume that the $\rho$ th class of $H^{n}$ is completely contained in $G$, and denote by $B^{\prime}$ the proper principal submatrix of $B$ obtained through deletion of the $\rho$ th row and column. Then there exists a vector $w$ such that $H^{n}-G$ has structure $B^{\prime}\langle\mathbf{w}\rangle$; and

$$
e_{G}=e\left(H^{n}\right)-e(G)-e\left(H^{n}-G\right) .
$$

(I) Determining an augmented graph structure. We shall prove that there exists a reduced augmentation $A^{\prime}$ of $A$ such that $A^{\prime}\left(m_{5}\right) \subseteq B(\infty)$. This will contradict (9.1) since, by (3.2), (9.2), and Lemma $5, g(B) \geqslant g\left(A^{\prime}\right) \geqslant g(A)+c$.

$\left(\mathrm{I}_{1}\right)$ We first prove the existence of positive constants $c^{\prime}=c^{\prime}(B), c^{\prime \prime}=c^{\prime \prime}(B)$ such that $H^{n}-G$ contains $c^{\prime} n$ vertices belonginig to the same class $C^{\prime}$ of $H^{n}-G$, each joined to $A\left[m_{2}\right]$ by at least $\left\{g(B)+c^{\prime \prime}\right\} m_{2}$ arcs. (While $c^{\prime \prime}$ appears to depend on $B$, Theorems 1 and 2 will imply the existence of $c^{\prime \prime}$ independent of $B$.) Let $B^{\prime \prime}$ be the principal submatrix of $B$ corresponding to classes of $H^{n}$ containing vertices of $G$. Since $\rho>m_{2}, B^{\prime \prime}$ must be a proper submatrix of the dense matrix $B$, so $g\left(B^{\prime \prime}\right)<$ $g(B)$. By (3.2) there must exist a constant $c^{\prime \prime}=c^{\prime \prime}(B)>0$ such that

$$
\begin{aligned}
e\left(H^{n}-G\right) & \leqslant \frac{1}{2} g\left(B^{\prime}\right)\left(n-m_{2} h\right)^{2}<\frac{1}{2}\left\{g(B)-2 c^{\prime \prime}\right\}\left(n-m_{2} h\right)^{2}, \\
e(G) & \leqslant \frac{1}{2} g\left(B^{\prime \prime}\right)\left(m_{2} h\right)^{2}<\frac{1}{2}\left\{g(B)-2 c^{\prime \prime}\right\}\left(m_{2} h\right)^{2},
\end{aligned}
$$

while, by (3.2),

$$
e\left(H^{n}\right)=\frac{1}{2} g(B) n^{2}+o\left(n^{2}\right) \text { as } n \rightarrow \infty .
$$

From (9.3) through (9.6) we obtain

$$
\begin{aligned}
e_{G} & >\frac{1}{2}\left\{2 c^{\prime \prime}+o(1)\right\} n^{2}+\left\{g(B)-2 c^{\prime \prime}\right\} m_{2} h\left(n-m_{2} h\right) \\
& >\left\{g(B)+2 c^{\prime \prime}+o(1)\right\} m_{2} h\left(n-m_{2} h\right) \text { as } n \rightarrow \infty \\
& >\left\{g(B)+c^{\prime \prime}\right\} m_{2} h\left(n-m_{2} h\right) \quad \text { for } n \text { sufficiently large. }
\end{aligned}
$$


Let $C^{\prime}$ be a $B^{\prime}$-class of $H^{n}-G$ whose vertices are each connected to vertices of $G$ by, on the average, the maximum total number of arcs, viz. by more than $\left\{g(B)+c^{\prime \prime}\right\} m_{2} h$. There exists a "small" positive constant $c^{\prime}=c^{\prime}(B)$ such that $\left|C^{\prime}\right|>c^{\prime} n$ for all $n$. All that we require is that $\left|C^{\prime}\right| \geqslant m_{5}$.

$\left(\mathrm{I}_{2}\right)$ The matrix $A^{\prime \prime} . A^{\prime \prime} \in \mathscr{M}_{r+1}$ is obtained from $A$ by adjoining an $(r+1)$ th row and column, defined as follows:

(i) where $C_{i}$ contains at least $m_{5}$ vertices each joined to and from vertices in $C^{\prime}$ by arcs in both directions, we put $a_{i, r+1}=a_{r+1, i}=2$;

(ii) where $C_{i}$ contains at least $m_{5}$ vertices joined to vertices in $C^{\prime}$ by single arcs directed towards $C^{\prime}$, but (i) does not hold, we put $a_{i, r+1}=2, a_{r+1, i}=0$;

(iii) where neither (i) nor (ii) holds, but where vertices of $C^{\prime}$ are joined to at least $m_{5}$ vertices of $C_{i}$ by single arcs directed away from $C^{\prime}$, we put $a_{i, r+1}=0$, $a_{r+1, i}=2$;

(iv) by Lemma 4 applied to $B$, one of the cases (i)-(iii) must hold for each $i$ $(i=1, \ldots, r)$;

(v) $a_{r+1, r+1}$ is defined to be zero.

Taking the appropriate $m_{5}$ vertices of $C^{\prime}$ and $m_{5}$ from each $C_{i}(i=1,2, \ldots, r)$ we obtain (for $n>m_{5} / c^{\prime}$ ) a subdigraph of $H^{n}$ of structure $A^{\prime \prime}\left\langle m_{5} \mathbf{e}\right\rangle$. Hence, for $A^{\prime}=D\left(A^{\prime \prime}\right), A^{\prime}\left(m_{5}\right) \subseteq H^{n}$. We must prove that $A^{\prime \prime}$ is an augmentation of $A$, i.e. that condition (6.1) is satisfied. When we now speak of $A\left[m_{2}\right]$ as a subdigraph of $G$ or of $H^{n}$, we intend a fixed one of the possible subdigraphs obtainable by selecting one vertex from each of the sets $\mathscr{I}_{x}$; only in subparagraph $\left(\mathrm{I}_{5}\right)$ will be require more than one vertex in any $\mathscr{I}_{x}$.

$\left(\mathrm{I}_{3}\right)$ Some vertex of any full $A$-class $C_{i}$ of $A\left[m_{2}\right]$ is joined to all vertices of $C^{\prime}$ by double arcs. Assume the contrary-that each vertex of $C^{\prime}$ is adjacent to $A\left[m_{2}\right]$ by at most one plus the valency in $A\left[m_{2}\right]$ of any vertex in $C_{i}$, which is $\left(1-a_{i i}\right)+$ the $i$ th component of $\hat{A} m_{2} \mathbf{u}$, i.e. $g(A) m_{2}$ arcs. But we chose $C^{\prime}$ so that the average would be at least $\left\{g(B)+c^{\prime \prime}\right\} m_{2}$ as $n \rightarrow \infty$.

$\left(\mathrm{I}_{4}\right)$ Suppose $a_{i i}=1, a_{r+1, i}+a_{i, r+1}<4$. by $\left(\mathrm{I}_{3}\right), C^{\prime}$ and $C$ cannot both be subsets of the same full class of $H^{n}$; hence $C^{\prime}$ is in an independent class of $H^{n}$, and vertices of $C_{i}$ are contained in distinct independent classes, $\bar{C}_{1}, \bar{C}_{2}, \ldots, \bar{C}_{m_{2}}$ of $H^{n}$. Let $C^{\prime \prime}$ be a set of $m_{5}$ vertices in $\bar{C}_{m_{2}}$. By Theorem 5 any vertex in $C^{\prime \prime}$ is adjacent with vertices of $G$ outside of $C_{i}$ by double arcs, with all but one vertex in $C_{i}$ by a single arc at least. Suppose that $a_{r+1, j}+a_{j, r+1}<4$, where $j \neq i$. Then the number of double arcs connecting a vertex of $C^{\prime}$ with $C_{j}$ would be less than $m_{5}$. By the maximality assumption on $C^{\prime}$, any vertex of $C^{\prime}$ would have to be connected with vertices of $C_{i}$ by at least $\left(m_{2}-1\right)+m_{2}-m_{5}$ arcs; there would certainly be $m_{5}$ vertices in $C_{i}$ connected to vertices of $C^{\prime}$ by double arcs, a contradiction. We may conclude that $a_{r+1, j}=a_{j, r+1}=2$ when $j \neq i$.

$\left(\mathrm{I}_{5}\right)$ Suppose $a_{i i}=1, a_{r+1, i}+a_{i, r+1}=2, a_{r+1, j}=a_{j, r+1}=2(j \neq i)$. By $\left(\mathrm{I}_{1}\right)$, some vertex $x \in C_{i}$ is adjacent by double arcs with every vertex of $C^{\prime}$. By replacing $C_{i}$ by $C^{\prime}$ and $m_{5}$ vertices in $\mathscr{I}_{x}$, and selecting $m_{5}$, we obtain a $B$-colourable graph $A_{1}\left(m_{4}\right)$, where $A_{1}$ is the splitting of the $i$ th row and column of $A$. This contradicts the hypothesis of (E). We must conclude that whenever $a_{i i}=1, a_{r+1, i}=a_{i, r+1}=2$. 
( $\left.\mathrm{I}_{6}\right)$ We form $A\left\langle m_{5} \mathbf{e}\right\rangle$ by selecting from each class $C_{i}$ the $m_{5}$ vertices having the highest adjacencies with a vertex of $C^{\prime}$. The number of such adjacencies is

$$
\frac{1}{2} \sum_{i=1}^{r}\left(a_{r+1, i}+a_{i, r+1}\right) m_{5} u_{i} \geqslant \frac{m_{5}}{m_{2}}\left\{g(A)+c^{\prime \prime}\right\} m_{2}, \quad \text { by }\left(\mathrm{I}_{1}\right) .
$$

By (6.1), $A^{\prime \prime}$ is an augmentation of $A$. Lemma 6 and (9.2) lead to a contradiction.

EXAMPLE. For use in a forthcoming example, and as an indication that the proof of Lemma 14 can be rewritten in constructive terms, we consider two specific simple examples. First we take $A_{1}=\left(\begin{array}{ll}0 & 2 \\ 2 & 0\end{array}\right)$, having density 1 , and no splittings. Up to equivalence, its dense augmentations are

$$
\left(\begin{array}{lll}
0 & 2 & 2 \\
2 & 0 & 2 \\
2 & 2 & 1
\end{array}\right), \quad\left(\begin{array}{lll}
0 & 2 & 2 \\
2 & 0 & 2 \\
2 & 2 & 0
\end{array}\right), \quad\left(\begin{array}{lll}
0 & 2 & 2 \\
2 & 0 & 2 \\
2 & 0 & 2
\end{array}\right),
$$

of densities $\frac{3}{2}, \frac{4}{3}, \frac{8}{7}$; it has one other augmentation-not dense-

$$
\left(\begin{array}{lll}
0 & 2 & 2 \\
2 & 0 & 2 \\
2 & 0 & 1
\end{array}\right)
$$

having density $\frac{4}{3}$ and maximal dense submatrix $\left(\begin{array}{ll}0 & 2 \\ 2 & 1\end{array}\right)$. So we may take $c_{A_{1}}=\frac{1}{14}$. For $m \geqslant m_{3}\left(A_{1}\right)=2$, every class of $A_{1}(m)$ is nonempty and contains $A_{1}\langle\mathbf{e}\rangle$; if $A_{1}\langle\mathbf{e}\rangle$ were $B$-colourable, so also would be all $A_{1}(n)$. We take $m_{A_{1}}=2$. $A_{1}$ is the only splitting of $A=(1)$; its augmentations, both dense, are $\left(\begin{array}{ll}1 & 2 \\ 2 & 0\end{array}\right),\left(\begin{array}{ll}1 & 2 \\ 2 & 1\end{array}\right)$, having densities $\frac{4}{3}, \frac{3}{2}$, respectively. We may take $c_{A}=\frac{1}{14}$. By Lemma 9 and the example of $\S 7, A_{1}$ is the only dense matrix $B$ such that $B \neq(1)$ and $1 \leqslant g(B)<\frac{15}{14}$. As $A(3)$ is not $A_{1}$-colourable, we may take $m_{A}$ to be 3 by the theory of (F). (The induction step is not required here. No attempt has been made to state best possible values for the various constants.)

10. Proofs of Theorems 1-3. For the purpose of this section we assume that the number $m_{A}$ of Lemma 14 is fixed for any $A$. We assert that the proof of Lemma 14 can be rewritten so that $m_{A}$ may be determined constructively.

Definition 15. Let $q=1$, and let $\alpha$ be any positive real number. $\mathscr{J}_{\alpha}=\left\{A\left(m_{A}\right)\right.$ : $A \in \mathscr{D}, \quad g(A)=\alpha\} . \quad \mathscr{K}_{\alpha}=\left\{A\langle\mathbf{e}\rangle: \quad A=\left(a_{i j}\right) \in \mathscr{D}, \quad g(A)>\alpha, \quad a_{i i}=0 \quad(i=\right.$ $1,2, \ldots)\}$.

LEMMA 15. (a) If $A$ has zero trace and $A\langle\mathbf{e}\rangle$ is B-colourable, then $g(A) \leqslant g(B)$.

(b) The mapping $f_{\alpha}$ onto $\mathscr{J}_{\alpha} \cup \mathscr{K}_{\alpha}$ defined by

$$
\begin{array}{ll}
A \mapsto A\left(m_{A}\right) & \text { if } g(A)=\alpha, \\
A \mapsto A\langle\mathbf{e}\rangle & \text { if } g(A)>\alpha \text { and } A \text { has zero trace }
\end{array}
$$

is one-to-one.

(c) If $g(A)=\alpha$, the only $A$-colourable digraph in $\mathscr{J}_{\alpha} \cup \mathscr{K}_{\alpha}$ is $A\left(m_{A}\right)$.

Proof. (a) Since $A$ has zero trace and $A\langle\mathbf{e}\rangle$ is $B$-colourable, $A \uparrow B$. We apply Lemma 5. 
(b) Suppose that $f_{\alpha}(A)=f_{\alpha}(B)$, hence $A\langle\mathbf{e}\rangle$ is $B$-colourable, $B\langle\mathbf{e}\rangle$ is $A$-colourable. If $g(A)>\alpha, A$ has zero trace; by (a), $B$ also has zero trace; from $A\langle\mathbf{e}\rangle=B\langle\mathbf{e}\rangle$ we may conclude that $A \approx B$. Similarly, if $g(B)>\alpha$. If $g(A)=\alpha=g(B), A\left(m_{A}\right)$ would be $B$-colourable, contradicting Lemma 14 .

(c) If $g(B)=\alpha$, then $f_{\alpha}(B)=B\left(m_{B}\right)$ cannot be $A$-colourable, by Lemma 14 applied to $B$. If $f_{\alpha}(B)=B\langle\mathbf{e}\rangle$ is $A$-colourable, where $g(B)>\alpha$ and $B$ has zero trace, then, by $(\mathrm{A}), g(B) \leqslant g(A)$, a contradiction.

Proof of TheOrem 1. (A) Let $\gamma>0$. We wish to prove $\mathscr{I}_{\gamma}$ finite. Application of Theorem A to $\mathscr{L}=\mathscr{J}_{\gamma} \cup \mathscr{K}_{\gamma}$ yields a matrix $U \in \mathscr{D}$ which is asymptotically extremal. If $g(U)>\gamma$, then, by Lemma 13, there exists $U^{\prime} \in \mathscr{M}$ with zero trace such that $g\left(U^{\prime}\right)>g(U)-\frac{1}{2}\{g(U)-\gamma\}>\gamma$, and no member of $\mathscr{L}$ is $U^{\prime}$-colourable; but $U^{\prime}\langle\mathbf{e}\rangle \in \mathscr{K}_{\gamma}$, a contradiction. Nor could $g(U)=\gamma$, for then $U\left(m_{U}\right)$ would be $U$-colourable, and $U$ could not be asymptotically extremal. It follows that $g(U)<\gamma$. Define $\alpha=\gamma-g(U)$. Then

$$
\operatorname{ex}\left(n, \mathscr{J}_{\gamma} \cup \mathscr{K}_{\gamma}\right)=\frac{1}{2}(\gamma-\alpha) n^{2}+o\left(n^{2}\right) \text { as } n \rightarrow \infty \text {. }
$$

(B) By Lemma $11 \mathscr{A}(\{\varnothing\}, \gamma \bmod \mathscr{L})$ is finite. Suppose $B$ is a matrix in the scheme having reduced augmentation $B^{\prime}$ such that $B^{\prime}(\infty)$ contains a prohibited subdigraph (in $\mathscr{J}_{\gamma} \cup \mathscr{K}_{\gamma}$ ): Fix one such subdigraph $L\left(B^{\prime}\right)$. We denote by $\mathscr{L}_{\gamma}$ the family of these $L\left(B^{\prime}\right)$ as $B^{\prime}$ ranges over all $B$. Since $\Lambda_{\gamma}$ is finite, and since (by Lemma 15) dense matrices of density $\gamma$ are equinumerous with members of $\mathscr{J}_{\gamma}$, Theorem 1 will follow from the finiteness of $\mathscr{J}_{\gamma}-\mathscr{L}_{\gamma}$.

(C) Let $A\left(m_{A}\right) \in \mathscr{J}_{\gamma}-\mathscr{L}_{\gamma}$. Since $g(A)>\gamma-\alpha, A(\infty)$ must contain a prohibited subdigraph in $\mathscr{J}_{\gamma} \cup \mathscr{K}_{\gamma}$; by Lemma 15 this is uniquely $A\left(m_{A}\right)$. Among all matrices $B$ in the finite scheme such that $B \uparrow A$, select one for which $g(B)$ is maximal; $g(B) \leqslant \gamma-\alpha<\gamma=g(A)$. By Lemma 9 there exists a reduced augmentation $B^{\prime}$ of $B$ such that $B^{\prime} \uparrow A . B^{\prime}$ cannot belong to the scheme, so $B^{\prime}(\infty)$ (and hence also $A(\infty)$ ) must contain a subdigraph in $\mathscr{L}_{\gamma}$. By Lemma 15 again, this is $A\left(m_{A}\right)$, which we have assumed not to be in $\mathscr{L}_{\gamma}$. We conclude that $\mathscr{J}_{\gamma} \subseteq \mathscr{L}_{\gamma}$.

EXAMPLE. $\mathscr{J}_{\gamma}$ may be properly contained in $\mathscr{L}_{\gamma}$. Let $q=1, \gamma=\frac{2}{3}$. There are (up to equivalence) two matrices of density $\frac{2}{3}$ :

$$
A^{\prime}=\left(\begin{array}{lll}
0 & 0 & 0 \\
2 & 0 & 0 \\
2 & 2 & 0
\end{array}\right) \quad \text { and } A^{\prime \prime}=\left(\begin{array}{lll}
0 & 0 & 2 \\
2 & 0 & 0 \\
0 & 2 & 0
\end{array}\right)
$$

having matrix digraphs containing, respectively, only transitive triangles and only cyclic triangles. Let $\mathscr{L}=\left\{A^{\prime}(3), A^{\prime \prime}(3)\right\}$. The matrix $U=\left(\begin{array}{ll}0 & 2 \\ 0 & 0\end{array}\right)$ yields an asymptotically extremal sequence for $\mathscr{L}$ (indeed, extremal). For no $U(n)$ contains a triangle; and $g(U)=\frac{1}{2}$. But, by the Erdös-Stone Theorem [2,9] for any $\varepsilon>0$, any fixed positive integer $m$, and sufficiently large $n$, any $G^{n}$ having $\left(\frac{1}{4}+\varepsilon\right) n^{2}$ arcs must contain a matrix digraph $A^{\prime}(m)$ or $A^{\prime \prime}(m)$. Up to equivalence, $\mathscr{K}_{2 / 3}$ consists of all graphs $A\langle\mathbf{e}\rangle$ where $A$ is dense and contains one of the following as a principal dense submatrix:

$$
A^{(3)}=\left(\begin{array}{ll}
0 & 2 \\
2 & 0
\end{array}\right), \quad A^{(4)}=\left(\begin{array}{llll}
0 & 2 & 2 & 2 \\
0 & 0 & 2 & 2 \\
0 & 0 & 0 & 2 \\
0 & 0 & 0 & 0
\end{array}\right) \text {. }
$$


The first level in $\mathscr{A}\left(\{\varnothing\}, \frac{2}{3} \bmod \mathscr{L}\right)$ contains only $(0)$. Augmenting by $\geqslant \frac{2}{3}$ modulo $\mathscr{L}$ we obtain no other matrices. But the digraph $L(B)$ which we choose in order to exclude the augmentation matrix $\left(\begin{array}{ll}0 & 2 \\ 2 & 0\end{array}\right)$ from the scheme cannot be either of the members of $\mathscr{J}_{2 / 3}$. However, both members of $\mathscr{J}_{2 / 3}$ may appear in $\mathscr{L}_{2 / 3}$; for example, $L((1))$, chosen for the augmentation of $\varnothing$, could be $A^{\prime}\left(m_{A^{\prime}}\right)$ (but it could also be $\left.A^{(4)}\langle\mathbf{e}\rangle\right)$. $\mathscr{L}\left(\left(\begin{array}{ll}0 & 2 \\ 2 & 1\end{array}\right)\right)$, from the augmentation of $(0)$, could be $A^{\prime \prime}\left(m_{A^{\prime \prime}}\right)$.

Proof of TheOREM 2. Let $\gamma$ be any positive real number, and define $\mathscr{L}$ and $\mathscr{L}_{\gamma}$ as in the proof of Theorem 1. Let $A$ be dense, $g(A)>\gamma$; let $B$ be a matrix in $\mathscr{A}\left(\{\varnothing\}, \gamma,\left\{A^{n}\right\} \bmod \mathscr{L}\right)$ such that $B \uparrow A$ and $g(B)$ is maximal. If $g(B)=\gamma$, $B\left(m_{B}\right) \in \mathscr{J}_{\gamma} \subseteq \mathscr{L}$ would be $B$-colourable. If $g(B)<\gamma$, then, by Lemma 9 , there exists a reduced augmentation $B^{\prime}$ of $B$ by $\geqslant g(A)$ such that $B^{\prime} \uparrow A$. $B^{\prime}$ is not in the scheme, so there must exist as an $A$-colourable digraph either (i) $L\left(B^{\prime}\right)=C\left(m_{C}\right) \in$ $\mathscr{J}_{\gamma} \cap \mathscr{L}_{\gamma}, g(C)=\gamma$; or (ii) $L\left(B^{\prime}\right)=C\langle\mathbf{e}\rangle \in \mathscr{J}_{\gamma} \cap \mathscr{L}_{\gamma}, g(C)>\gamma$; and there also remains the possibility that (iii) $g(A)>g(B)>\gamma$. In (i) $g(A) \geqslant g(C)+c_{C}=\gamma+c_{C}$ (Lemma 14); in (ii) $g(A) \geqslant g(C)>\gamma$ (Lemma 15). Define $c_{3}=\operatorname{Min}\left\{\gamma+c_{C}\right.$ : $\left.C\left(m_{C}\right) \in \mathscr{J}_{\gamma} \cap \mathscr{L}_{\gamma}\right\}, c_{4}=\operatorname{Min}\left\{g(C): C\langle\mathbf{e}\rangle \in \mathscr{J}_{\gamma} \cap \mathscr{L}_{\gamma}\right\}, c_{5}=\operatorname{Min}\{g(B): g(B)>$ $\left.\gamma, B \in \mathscr{A}\left(\{\varnothing\}, \gamma,\left\{A^{n}\right\} \bmod \mathscr{L}\right)\right\}$. The three sets to be minimized are all finite, by Theorem 1, and all their elements are greater than $\gamma$. We can conclude that no dense matrix $A$ has the property that

$$
\gamma<g(A)<\operatorname{Min}\left\{c_{3}, c_{4}, c_{5}\right\} .
$$

Thus the set of densities is well ordered.

Proof of Theorem 3. Let $q=1$, and let $\mathscr{L}$ be an infinite family of digraphs. For each positive integer $p$ let $\mathscr{L}^{(p)}$ denote the set of members of $\mathscr{L}$ having at most $p$ vertices. By Theorem A there exists for each $p$ a dense matrix $A^{(p)}$ which is asymptotically extremal for $\mathscr{L}^{(p)}$. Consequently

$$
\lim _{n \rightarrow \infty} \frac{\operatorname{ex}(n, \mathscr{L})}{\left(\begin{array}{l}
n \\
2
\end{array}\right)}=\lim _{p \rightarrow \infty} \lim _{n \rightarrow \infty} \frac{\operatorname{ex}\left(n, \mathscr{L}^{(p)}\right)}{\left(\begin{array}{l}
n \\
2
\end{array}\right)}=2 \lim _{p \rightarrow \infty} g\left(A^{(p)}\right)=2 g\left(A^{(p)}\right)
$$

for sufficiently large $p$, by Theorem 2 and the fact that $\operatorname{ex}\left(n, \mathscr{L}^{(p)}\right) /\left(\begin{array}{c}n \\ 2\end{array}\right)$ is monotonely nonincreasing in both $n$ and $p$. Any matrix asymptotically extremal for $\mathscr{L}$ will surely be asymptotically extremal for $\mathscr{L}^{(p)}$ for sufficiently large $p$. By Theorem $i$ there are finitely many matrices $A$ such that $g(A)=\lim _{n \rightarrow \infty} 2\left(\operatorname{ex}(n, \mathscr{L}) /\left(\begin{array}{l}n \\ 2\end{array}\right)\right)$. For any such $A$ which is not asymptotically extremal for $\mathscr{L}$, there will exist a positive integer $p_{A}$ such that some $L \in \mathscr{L}^{(p)}$ is $A$-colourable when $p>p_{A}$. Taking $p$ greater than all such $p_{A}$, we obtain $\mathscr{L}^{*}=\mathscr{L}^{(p)}$ having the same asymptotically extremal matrices as $\mathscr{L}$.

\section{Proof of Theorem 4.}

Introduction to the algorithm. (A) Let $q=1$, and let $\mathscr{L}$ be a given family of digraphs. We describe a method that must eventually yield all asymptotically extremal matrices $A$. We do not consider the efficiency of the procedure.

(B) There exists an enumeration of all matrices $A \in \mathscr{M}$. This cannot list the matrices in increasing order of their densities, since, notwithstanding Theorems 1 and 2, the set of densities has infinitely many limit points (the least is 1 , which is the 
limit of the densities of triangular matrices with 2's above the main diagonal and 0's elsewhere). For any $A \in \mathscr{M}_{r}$, there exists a bound-depending only on $r$-on the number of integer arithmetic and logical operations which must be performed to determine its density, and whether it is dense (cf. Lemma 1). We leave it to the reader to verify that Lemmas 7 and 9 may be rewritten in constructive versions, so that, in Lemma 14, both $c_{A}$ and $m_{A}$ can be determined in a bounded number of steps. We describe below an algorithm for determining, for a given $A \in \mathscr{D}_{\text {, }}$, whether or not $A$ is asymptotically extremal for $\mathscr{L}$.

(C) A dense matrix $A$ will be an end-matrix if it is a reduction of a restricted type of augmentation defined below, and if, in addition, each of its reduced augmentations $A^{\prime}$ of this restricted type has the property that some $L \in \mathscr{L}$ is $A^{\prime}$-colourable. There exists a recursive function $\bar{m}=\bar{m}_{A}$ defined on the end-matrices $A$ such that if (i) $B$ is dense, (ii) $g(B) \geqslant g(A)$, (iii) $B \neq A$, (iv) $A\left(\bar{m}_{A}\right)$ is $B$-colourable, then some $L \in \mathscr{L}$ is $B$-colourable. (Let $m$ denote a sufficiently large integer that, for each of the-finitely many-augmentations $A^{\prime}$ of $A, A^{\prime}(m)$ contains some $L \in \mathscr{L}$. By Lemma 14, conditions (i)-(iv) ensure that $g(B) \geqslant g(A)+c_{A}$; by Lemma 3 , all valencies in $B(n)$-for $n$ sufficiently large-exceed $\left\{g(A)+c_{A} / 2\right\}$. Applying Lemma 7, we conclude that $B(n)$ contains an $A^{\prime}(m)$, which, in turn, contains some $L \in \mathscr{L}$. Our proof requires constructive versions of Lemmas $3,7,14$.)

(D) We construct recursively a finite subscheme $\mathscr{A}_{\mathscr{L}}$ of $\mathscr{A}(\{\varnothing\}, \gamma \bmod \mathscr{L})$, in which we augment "by" progressively larger constants. Each level will require a finite number of decisions of the type "does $\mathscr{L}$ contain a $B$-colourable digraph". Suppose that $\mathscr{A}_{\mathscr{L}}$ has been defined for $\mathscr{S}_{j-1}, j>0$. The construction process involves (i) determination of the finitely many matrices of $\mathscr{S}_{j}$; (ii) scanning of each of these to determine which matrices in $\mathscr{S}_{j-1}$ are end-vertices; (iii) determination, for each end-vertex $A$ in $\mathscr{S}_{j-1}$, of $\bar{m}_{A}$ and $c_{A}$; (iv) determination of the maximum, $\gamma_{j}$, of the densities of all matrices then in the scheme. The process begins with $\varnothing$, having density $-\infty$, and reduced augmentations (0) and (1).

Description of the algorithm. (I) Let $j=0 . \mathscr{S}_{0}=\{\varnothing\} \cdot \gamma_{0}=-\infty$. Set $j=1$ and go to (II).

(II) Determine all reduced augmentations $A^{\prime}$ of each matrix $A$ of $\mathscr{S}_{j-1}$ by $\geqslant \gamma_{j-1}$. Exclude, however, all $A^{\prime}$ such that either (a) some $L \in \mathscr{L}$ is $A^{\prime}$-colourable; or (b) some digraph $C\left(\bar{m}_{C}\right)$ is $A^{\prime}$-colourable, where $C \approx A^{\prime}$ and $C$ is an end-vertex of the scheme in some level up to but not including the $(j-1)$ th. These augmentations, excluding those which are already present in the scheme, constitute the members of $\mathscr{S}_{j}$, and an arc is directed from each $A$ in $\mathscr{S}_{j-1}$ to its reduced augmentations (unless such arc is already present from processing earlier levels); an arc may even be directed to a matrix in a lower level, but only if all intervening values of $\gamma_{k}$ are equal. Go to (III).

(III) For every $A$ in $\mathscr{S}_{j-1}$, if every (direct) augmentation $A^{\prime}$ of $A$ has been excluded, designate $A$ an end-vertex of the scheme. Go to (IV).

(IV) Determine, for the end-vertices $A$ in $\mathscr{S}_{j-1}$, the constants $\bar{m}_{A}$ (defined above), and $c_{A}$ (defined in Lemma 14). As seen in (C), there must exist, for any dense matrix $B$ such that $g(A) \leqslant g(B)$ and $A\left(\bar{m}_{A}\right)$ is $B$-colourable, a $B$-colourable $L \in \mathscr{L}$. Set $\gamma_{j}$ 
equal to the maximum of $\gamma_{j-1}$ and of the densities of all matrices in $\mathscr{S}_{j}$. If all matrices in $\mathscr{S}_{j-1}$ are end-matrices, go to $(\mathrm{V})$; otherwise replace $j$ by $j+1$ and go to (II).

(V) End-matrices having maximum density will be the extremal matrices for $\mathscr{L}$.

REMARKS. (1) Before commencing verification of the algorithm, we attempt to explain and to illustrate its operation.

(2) The careful reader may wonder why condition (b) of (II) is needed. While we augment all $A$ in $\mathscr{S}_{j}$ by at least the maximum density of members of the level, the densities of the augmentations obtained need not be greater than-or even equal to - that maximum; thus it may happen that some such augmentation $A^{\prime}$ is such that no $L \in \mathscr{L}$ is $A^{\prime}$-colourable, yet $A^{\prime}$ contains some $C\left(\bar{m}_{C}\right)$. Our proof will show that such an $A^{\prime}$ is not required in the procedure of determining the desired matrices; and -if we did not exclude it-might render the process infinite (cf. the example below).

(3) The example provided below is intended to illustrate the various stages of the algorithm-not to demonstrate its effectiveness in resolving the extremal problem for the given digraph. The exact solution is known in this case: $\operatorname{ex}(n, L)=2\left[n^{2} / 4\right]$; indeed, $\operatorname{EX}(n, L)$ is also known (cf. [8, Theorem (6.1), (6.5)]). Notwithstanding the extended length of the discussion, we have used ad hoc arguments at several stages to abbreviate the procedure.

EXAMPLE. Let $\mathscr{L}$ consist of one 3-vertex digraph $L$-having two "double" arcs and one "single" arc. $\mathscr{S}_{0}=\{\varnothing\}, \gamma_{0}=-\infty . \mathscr{S}_{1}=\{(0),(1)\}, \gamma_{1}=1$; neither $(0)(\infty)$ nor $(1)(\infty)$ contains $L$, so $\varnothing$ is not an end-matrix. No augmentation of (1) survives, as both "contain" $L$. Up to equivalence, the only members of $\mathscr{S}_{2}$ are $\left(\begin{array}{ll}0 & 0 \\ 2 & 0\end{array}\right),\left(\begin{array}{ll}0 & 2 \\ 2 & 0\end{array}\right)$, having densities $\frac{1}{2}, 1$, respectively. Here $\gamma_{2}=1$, and (1) is the only end-matrix in $\mathscr{S}_{1}$. (At this point we would normally have to determine $\bar{m}_{(1)}$ and $c_{(1)}$. By the example of $\S 9$, we may take these constants to be 3 and $\frac{1}{14}$ respectively. In fact, $A(3)$ cannot be $B$-colourable when $g(B)=1$ and $B \neq A=(1)$, since then $B=\left(\begin{array}{ll}0 & 2 \\ 2 & 0\end{array}\right)$, and $B(\infty)$ has no triangles.) Augmenting $\left(\begin{array}{ll}0 & 0 \\ 2 & 0\end{array}\right)$ and reducing yields (up to equivalence) (1), $\left(\begin{array}{ll}0 & 2 \\ 2 & 0\end{array}\right)$, and the following 5 matrices:

$$
\left(\begin{array}{lll}
0 & 0 & 0 \\
2 & 0 & 0 \\
2 & 2 & 0
\end{array}\right),\left(\begin{array}{lll}
0 & 0 & 2 \\
2 & 0 & 0 \\
0 & 2 & 0
\end{array}\right),\left(\begin{array}{lll}
0 & 0 & 2 \\
2 & 0 & 2 \\
2 & 2 & 0
\end{array}\right),\left(\begin{array}{lll}
0 & 0 & 2 \\
2 & 0 & 2 \\
2 & 2 & 1
\end{array}\right),\left(\begin{array}{ll}
0 & 2 \\
2 & 1
\end{array}\right),
$$

having densities $\frac{2}{3}, \frac{2}{3}, \frac{8}{7}, \frac{7}{5}, \frac{4}{3}$, respectively; of these, the last three "contain" $L$. Augmenting $\left(\begin{array}{ll}0 & 2 \\ 2 & 0\end{array}\right)$ yields, in addition to

$$
\left(\begin{array}{lll}
0 & 0 & 2 \\
2 & 0 & 2 \\
2 & 2 & 0
\end{array}\right) \text { and }\left(\begin{array}{ll}
0 & 2 \\
2 & 1
\end{array}\right)
$$

the matrices

$$
\left(\begin{array}{lll}
0 & 2 & 2 \\
2 & 0 & 2 \\
2 & 2 & 0
\end{array}\right) \text { and }\left(\begin{array}{lll}
0 & 2 & 2 \\
2 & 0 & 2 \\
2 & 2 & 1
\end{array}\right)
$$


having densities $\frac{4}{3}$ and $\frac{3}{2}$ respectively, and both "containing" $L$. Thus in $\mathscr{S}_{2}, A_{1}=\left(\begin{array}{ll}0 & 2 \\ 2 & 0\end{array}\right)$ is an end-vertex, and $\left(\begin{array}{ll}0 & 2 \\ 0 & 0\end{array}\right)$ is not. (By earlier computations, the only $B$ we need consider is (1). $A_{1}(2)$ is not $B$-colourable. So we may take $\bar{m}_{A_{1}}=2$.) We augment

$$
\left(\begin{array}{lll}
0 & 0 & 0 \\
2 & 0 & 0 \\
2 & 2 & 0
\end{array}\right) \text { and }\left(\begin{array}{lll}
0 & 0 & 2 \\
2 & 0 & 0 \\
0 & 2 & 0
\end{array}\right)
$$

by $\geqslant 1$. Reductions of augmentations not containing $L$ and not already in the scheme will be matrices associated with the various tournaments on four vertices, and their densities will be $\frac{3}{4}$. The process may be repeated. Without (II)(b), it would produce an infinite sequence of levels, containing the matrices associated with tournaments with increasing numbers of vertices, and all having density less than that of (1). However, sufficiently large tournaments must contain a transitive subtournament on $\bar{m}_{(1)}$ vertices, and the procedure must terminate. The matrices yielding asymptotically extremal sequences will then be (1) and $\left(\begin{array}{ll}0 & 2 \\ 2 & 0\end{array}\right)$.

Verification of the algorithm. (A) Let $U \in \mathscr{D}_{r}$ be an asymptotically extremal matrix for $\mathscr{L}$. Define $\gamma=g(U)=2 \lim _{n \rightarrow \infty}\left(\operatorname{ex}(n, \mathscr{L}) / n^{2}\right)$. By Lemma 9 there exists a nested sequence of principal dense submatrices $\varnothing=A_{0}, A_{1}, \ldots, A_{r}=U$, where $A_{i}$ is an augmentation of $A_{i-1}$ by $\geqslant \gamma>g\left(A_{i-1}\right)$ without reduction; we prove by induction that $A_{i}$ is in $\mathscr{A}_{\mathscr{L}}(i=1,2, \ldots, r)$. By definition, $\varnothing \in \mathscr{S}_{0}$. Since no $L \in \mathscr{L}$ is $U$-colourable, and the sequence is nested, no such $L$ is $A_{i}$-colourable, so $A_{i+1}$ will not be excluded in (II) by virtue of condition (a). We assert that no $C\left(\bar{m}_{C}\right)$ is $A_{i+1}$-colourable, where $C$ is an end-vertex of an earlier level of the scheme; for this would entail that $C\left(\bar{m}_{C}\right)$ be $U$-colourable, and so, by definition of $\bar{m}, U \approx C$. From this contradiction we conclude that $U$ belongs to the scheme $\mathscr{A}_{\mathscr{L}}$.

(B) By (3.2) and II(a), no matrix in $\mathscr{A}_{\mathscr{L}}$ can have density exceeding $\gamma$.

(C) We prove that for any $\mathscr{L}$-even infinite $\mathscr{L}-\mathscr{A}_{\mathscr{L}}$ can have only finitely many nonempty levels. We know by Theorem 1 that only finitely many dense matrices have density $\gamma$. Among these are the extremal matrices, say $U_{1}, U_{2}, \ldots, U_{t}$, which, by (A), all figure in the scheme, each as end-matrix. Let $j_{0}$ denote the highest level containing one of these matrices. By (B) above, $\gamma_{j}=\gamma$ for $j \geqslant j_{0}$. Let $\mathscr{L}^{*}=\mathscr{L} \cup$ $\left\{U_{k}\left(\bar{m}_{U_{k}}\right): k=1,2, \ldots, t\right\}$. By Theorem A there exists a dense matrix $V$ extremal for $\mathscr{L}^{*}$. $U_{k}\left(\bar{m}_{U_{k}}\right)$ cannot be $V$-colourable, so $V$ is distinct from $U_{k}(k=1,2, \ldots, t)$; since $\mathscr{L} \subseteq \mathscr{L}^{*}, g(V)<\gamma$. By II(b) none of the matrices above the $j_{0}$ th level can have density exceeding $g(V)$. As we are augmenting by $\gamma>g(V)$, we know from Lemma 7 that there can be only finitely many levels beyond the $j_{0}$ th.

(D) In the highest level all matrices will be end-matrices. Any matrix in $\mathscr{A}_{\mathscr{L}}$ having the maximum density $\gamma$ must be an end matrix, and must be one of $U_{1}, U_{2}, \ldots, U_{t}$; and, as observed earlier, each of $U_{1}, U_{2}, \ldots, U_{t}$ is an end-matrix.

12. Multigraphs. Our results for digraphs imply multigraph analogues. To distinguish multigraph versions from digraph versions in this context, we shall superimpose a circumflex ^ or an arrow $\rightarrow$ over certain symbols; in particular, $\overrightarrow{\mathrm{ex}}$ and $\overrightarrow{\mathrm{EX}}$ will denote the functions denoted by ex and EX in $\$ \$ 2-11$. That this use of ${ }^{\wedge}$ is consistent with Definition 7 will be shown below. 
Let $q$ be a fixed positive integer. We may relate multigraph extremal problems with odd maximum edge multiplicity $\hat{q}=2 q-1$ to problems where $\hat{q}$ is even by interpreting the former as having maximum edge multiplicity $2 q$, and adjoining to the family of prohibited multigraphs a multigraph $G_{0}$ having $2 q$ parallel edges connecting 2 vertices. The extremal problem for $\hat{q}=2 q-1$ and family $\mathscr{G}$ is equivalent to that for $\hat{q}=2 q$ and family $\mathscr{G} \cup\left\{G_{0}\right\}$.

Definition 16. (a) The underlying multigraph $\hat{L}$ of a digraph $L$ is obtained by suppressing orientations. The multiplicity of an edge will be the sum of the multiplicities of the arcs joining its ends with either orientation ${ }^{10}$. If $\mathscr{L}$ is a family of digraphs, $\hat{\mathscr{L}}=\{\hat{L}: L \in \mathscr{L}\}$.

(b) A (regular) orientation of a multigraph $G$ is any digraph $L$ such that $G=\hat{L}$. (An essential constraint is that arcs in either direction must not have multiplicity exceeding q.) The set of orientations of $G$ will be denoted by $\vec{G} ; \cup\{\vec{G}: G \in \mathscr{G}\}$ will be denoted by $\overrightarrow{\mathscr{G}}$.

Proposition. Let $\hat{q}=2 q$. Let $\mathscr{G}$ be a given family of multigraphs. Then

$$
\widehat{\widehat{\mathrm{EX}}(n, \mathscr{G})}=\overrightarrow{\mathrm{EX}}(n, \overrightarrow{\mathscr{G}}) .
$$

A multigraph $G^{n}$ is extremal for $\mathscr{G}$ if and only if it admits a regular orientation $S^{n}$ which is extremal for $\overrightarrow{\mathscr{G}}$; moreover, if $G^{n}$ is extremal for $\mathscr{G}$, then every regular orientation $S^{n}$ is extremal for $\overrightarrow{\mathscr{G}}$.

The preceding permits the reduction of each multigraph extremal problem (with even multiplicity $\hat{q}$ ) to a corresponding digraph problem.

Definition 17. Matrix multigraphs. Let $\hat{A}=\left(a_{i j}\right)$ be a symmetric $r \times r$ matrix, for which $a_{i j}=0,1, \ldots$, or $2 q$ if $i \neq j$; and $a_{i i}=0,1, \ldots$, or $2 q-1$. For every partition $n=x_{1}+x_{2}+\cdots+x_{r}$ into nonnegative integers we define a multigraph $\hat{A}\langle\mathbf{x}\rangle$ as follows: (i) $n$ vertices are divided into $r$ classes $C_{1}, C_{2}, \ldots, C_{r}$, where $\left|C_{i}\right|=x_{i}$; (ii) every vertex of $C_{i}$ is joined to every (distinct) vertex of $C_{j}$ by $a_{i j}$ edges $(i, j=1,2, \ldots, r)$. The set of matrices defined above may be denoted by $\hat{\mathcal{M}}_{r}$.

For any $A \in \mathscr{M}_{r}$, the matrix $\hat{A}$ of Definition 7 satisfies the conditions of Definition 17. Conversely, for every matrix $\hat{A}$ satisfying Definition 17 , there exist $A \in \mathscr{M}_{r}$ such that $A+A^{*}=2 \hat{A}$. $A$ and $\hat{A}$ give rise to the same quadratic form (cf. Lemma 1). Density may be defined in the obvious way. Consequently, Theorem 1 for $q=1$ impliles an unoriented analogue for $\hat{q}=2$; likewise Theorem 2 .

THEOREM $\hat{1}$. Let $\hat{q}=2$. For any $\gamma>0$ there exist only finitely many symmetric dense matrices $A \in \hat{\mathscr{M}}$ such that $g(\hat{A})=\gamma$.

TheOREM $\hat{2}$. Let $q=2$. The set $\{\gamma: \gamma=g(\hat{A}), A \in \mathscr{D}\}$ is well ordered.

We shall not work systematically through all results for digraphs: analogues suggest themselves without surprises. We have proved a matrix analogue of Theorem $A$ in [3], announced one of Theorem B in [6], and stated other results in [4]. A

\footnotetext{
${ }^{10}$ We do not associate (as is sometimes the case, cf. [7]) pairs consisting of one arc in either direction with a single edge.
} 
multigraph analogue of Theorem 3 holds:

THEOREM 3. Let $q=2$. For every infinite family $\mathscr{G}$ of prohibited submultigraphs there exists a finite subfamily $\mathscr{G} * \subseteq \mathscr{G}$ for which

$$
\widehat{\operatorname{ex}}(n, \mathscr{G})=\widehat{\operatorname{ex}}\left(n, \mathscr{G}^{*}\right)+o\left(n^{2}\right) \text { as } n \rightarrow \infty \text {, }
$$

and such that any dense matrix $A \in \hat{\mathscr{M}}$ is asymptotically extremal for $\mathscr{G}$ if and only if it is asymptotically extremal for $\mathscr{G} *$.

Proof. By Theorem 3 there exists a finite set of digraphs $\mathscr{L} \subseteq \overrightarrow{\mathscr{G}}$ such that

$$
\begin{aligned}
\overrightarrow{\operatorname{ex}}(n, \mathscr{L}) & =\overrightarrow{\operatorname{ex}}(n, \overrightarrow{\mathscr{G}})+o\left(n^{2}\right) \\
& =\widehat{\operatorname{ex}}(n, \mathscr{G})+o\left(n^{2}\right) \text { as } n \rightarrow \infty,
\end{aligned}
$$

by (12.1). Since $\mathscr{L} \subseteq \overrightarrow{\hat{\mathscr{L}}} \subseteq \overrightarrow{\mathscr{G}}$,

$$
\overrightarrow{\operatorname{ex}}(n, \mathscr{L}) \geqslant \overrightarrow{\operatorname{ex}}(n, \overrightarrow{\hat{\mathscr{L}}}) \geqslant \overrightarrow{\operatorname{ex}}(n, \overrightarrow{\mathscr{G}}) \text {. }
$$

By (12.3), (12.1),

$$
\widehat{\operatorname{ex}}(n, \overrightarrow{\mathscr{L}})=\widehat{\operatorname{ex}}(n, \mathscr{G})+o\left(n^{2}\right) \text { as } n \rightarrow \infty \text {. }
$$

If $\{\hat{A}(n)\}$ is an asymptotically extremal sequence for $\mathscr{G}$, then, by (12.4), the sequence is surely asymptotically extremal for $\hat{\mathscr{L}}$, which is a subset. If $\hat{A}$ is extremal for $\hat{\mathscr{L}}$, any matrix $A$ such that $\hat{A}=\frac{1}{2}\left(A+A^{*}\right)$ is extremal for $\mathscr{L}$, hence for $\overrightarrow{\mathscr{G}}$, and $\hat{A}$ is extremal for $\hat{\overrightarrow{\mathscr{G}}}=\mathscr{G}$. We take $\hat{\mathscr{L}}$ as $\mathscr{G}^{*}$.

Theorem 4 also particularizes to multigraphs with $\hat{q}=2$. It suffices to work with $\overrightarrow{\mathscr{G}}$ and to ignore nonsymmetric matrices produced by the algorithm.

\section{REFERENCES}

1. A. Aho, J. E. Hopcroft and J. D. Ullman, The design and analysis of computer algorithms, Addison-Wesley, Reading, Mass., 1974. ISBN 0-201-00029-6. MR 54 \#1706.

2. B. Bollobás, Extremal graph theory, Academic Press, London, 1978. ISBN 0-12-111750-2. MR 80a $\# 05120$.

3. W. G. Brown, P. Erdös, and M. Simonovits, Extremal problems for directed graphs, J. Combin. Theory Ser. B 15 (1973), 77-93. MR 52 \$7952.

4. Multigraph extremal problems, Colloques Internationaux C.N.R.S. 260: Problèmes combinatoires et théorie des graphes, Proc. Colloq., Orsay, 1976 (Editions C.N.R.S., Paris, 1978). ISBN 2-222-02070-0, 63-66.

5. . Multigraph extremal problems, preprint, 1975.

6. Inverse extremal digraph problems, Colloq. Math. Soc. János Bolyai 37, Finite and Infinite Sets, Eger (Hungary), 1981, Akad. Kiadó, Budapest, 1985, pp. 119-156.

7. W. G. Brown and M. Simonovits, Digraph extremal problems, hypergraph extremal problems, and the densities of graph structures, Discrete Math. 48 (1984), 147-162.

8. W. G. Brown and F. Harary, Extremal digraphs, Combinatorial Theory and its Applications, Colloq. Math. Soc. János Bolyai 4 (1970), I 135-198. MR 45 \#576.

9. P. Erdös and M. Simonovits, A limit theorem in graph theorv, Studia Sci. Math. Hungar. 1 (1966), 51-57. MR 34 \#5702.

10. P. Erdös, Some recent results on extremal problems in graph theory (Results), Theory of Graphs: (International Symposium, Rome, 1966), Gordon \& Breach, New York and Dunod, Paris, 1967, pp. 118-123. MR 37 2634.

11. , On some new inequalities concerning extremal properties of graphs, Theory of Graphs (Proc. Colloq.. Tihany, 1966), Academic Press, New York and Akad. Kiadó, Budapest, 1968, pp. 77-81. MR 38 $\# 1026$. 
12. R. Häggkvist and C. Thomassen, On pancyclic digraphs, J. Combin. Theory Ser. B 20 (1976), 20-40. MR 52 \#10481.

13. Gy. Katona, T. Nemetz and M. Simonovits, On a problem of Turan in the theory of graphs, Mat. Lapok 15 (1964), 228-238. MR 30 \#2483.

14. T. S. Motzkin and E. G. Straus, Maxima for graphs and a new proof of a theorem of Turán, Canad. J. Math. 17 (1965), 533-540. MR 31 \#89.

15. K. B. Reid, Two applications of Turán's theorem to asymmetric digraphs, Combinatorial Structures and their Applications, Proc. Calgary Internat. Conference (Calgary, Alberta, 1969), Gordon \& Breach, New York, 1970, pp. 351-353. MR 42 \#861.

16. M. Simonovits, A method for solving problems in graph theory, stability problems, Theory of Graphs (Proc. Colloq. Tihany, 1966), Academic Press, New York and Akad. Kiadó, Budapest, 1968, pp. 279-319. MR 38 \#2056.

17. Extremal graph theory, Selected Topics in Graph Theory 2 (L. W. Beineke and R. J. Wilson, eds.), Academic Press, London, 1983, pp. 161-200, ISBN 0-12-086202-2.

18. P. Turán, Egy gráfelméleti szélsöérték feladatról, Mat. Fiz. Lapok 48 (1941), 436-452. MR 8, 284j.

19. On the theory of graphs, Colloq. Math.3 (1954), 19-30. MR 15, 976b.

20. A. Zykov, On some properties of linear complexes, Mat. Sb. 24 (1949), 163-188; Amer. Math. Soc. Transl. (1) 79 (1959). MR 11, 733h.

Department of Mathematics and Statistics, McGill University, Montreal, Québec, Canada H3A $2 \mathrm{~K} 6$

M.T.A. Matematikai Kutató Intezet, 1053 Reáltanoda U. 13-15, Budapest, Hungary

Eotvós Loránd University, 1088 Budapest, MÚzeum Krt. 6-8, ANalizis I. Tanszé, Budapest, HUNGARY 